\title{
CONVERGENT NUMERICAL SCHEME FOR SINGULAR STOCHASTIC CONTROL WITH STATE CONSTRAINTS IN A PORTFOLIO SELECTION PROBLEM*
}

\author{
AMARJIT BUDHIRAJA $^{\dagger}$ AND KEVIN ROSS ${ }^{\dagger}$
}

\begin{abstract}
We consider a singular stochastic control problem with state constraints that arises in problems of optimal consumption and investment under transaction costs. Numerical approximations for the value function using the Markov chain approximation method of Kushner and Dupuis are studied. The main result of the paper shows that the value function of the Markov decision problem (MDP) corresponding to the approximating controlled Markov chain converges to that of the original stochastic control problem as various parameters in the approximation approach suitable limits. All our convergence arguments are probabilistic; the main assumption that we make is that the value function be finite and continuous. In particular, uniqueness of the solutions of the associated HJB equations is neither needed nor available (in the generality under which the problem is considered). Specific features of the problem that make the convergence analysis nontrivial include unboundedness of the state and control space and the cost function; degeneracies in the dynamics; mixed boundary (Dirichlet-Neumann) conditions; and presence of both singular and absolutely continuous controls in the dynamics. Finally, schemes for computing the value function and optimal control policies for the MDP are presented and illustrated with a numerical study.
\end{abstract}

Key words. singular control, Hamilton-Jacobi-Bellman equations, portfolio selection, stochastic control, free boundary problem, Skorohod problem

AMS subject classifications. 60K25, 68M20, 90B22, 90B35, 60J70

DOI. $10.1137 / 050640515$

1. Introduction. Singular control is an important and challenging class of problems in stochastic control theory. Roughly speaking, by singular control we mean that the control terms in the dynamics of the state process need not be absolutely continuous with respect to the Lebesgue measure and are only required to have paths of bounded variation. The Hamilton-Jacobi-Bellman (HJB) equations for such problems, which are variational inequalities with gradient constraints, are typically quite hard to work with. Despite the fact that over the past 20 years there has been a significant development in the theory of weak and viscosity solutions of HJB equations for such diffusion control problems (cf. [5, 26, 4, 15, 22, 29]), the existence/uniqueness and regularity theory for this class of PDEs is not well understood. In view of the various applications in mathematical finance (cf. [11]) and stochastic networks (cf. [12]) that lead to singular control, it is particularly important to develop methods for numerical approximations for such control problems.

Over the last 30 years, Kushner, Dupuis, and coworkers (cf. [19] and references therein) have developed a powerful machinery, the so-called Markov chain approximation method, for a wide spectrum of computational problems in stochastic control theory. This probabilistic approach has two main advantages. First, approximation with a Markov chain allows one to use physical insights derived from the dynamics

* Received by the editors September 17, 2005; accepted for publication (in revised form) August 20, 2006; published electronically January 22, 2007. This research was supported in part by ARO grant W911NF-04-1-0230. This work was undertaken while the second author was at the University of North Carolina at Chapel Hill.

http://www.siam.org/journals/sicon/45-6/64051.html

${ }^{\dagger}$ Department of Statistics and Operations Research, University of North Carolina at Chapel Hill, 210 Smith Building, CB\# 3260, Chapel Hill, NC 25799-3260 (budhiraj@email.unc.edu, kjross@ email.unc.edu). 
of the controlled diffusion in obtaining a stable approximation scheme. Second, the Markov chain approximation method does not require the smoothness of the cost or value function, nor does it rely on the uniqueness properties of the associated HJB equations. This is a particularly significant advantage in problems where the PDE theory for the associated HJB equations is hard to tackle.

In this work we study a problem of optimal consumption and portfolio selection with proportional transaction costs that has been studied by several authors $[24,6$, $29,23,31]$. The basic problem can be described as follows. Consider a single investor who has two instruments available for investment: a risk-free asset such as a bank account, which pays a fixed interest rate $r>0$, and a risky asset, such as a stock, whose price evolution is modeled via a geometric Brownian motion with a mean value of return $b>r$ and constant volatility $\sigma>0$. We assume that the investor may buy or sell stock continuously over time in not necessarily integer valued quantities. The investor is assumed to consume wealth at some time-dependent rate $C(t)$, and without loss of generality we assume that the consumption is deducted from the bank account. The investor may instantaneously transfer money from the bank account to stock, and vice versa, by paying a proportional transaction cost; namely, there are $\lambda \in(0, \infty)$ and $\mu \in(0,1)$ such that the investor pays $\lambda$ times the amount moved from the bank account to stock as a transaction fee, and similarly, he pays $\mu$ times the amount moved from stock to the bank account as a transaction fee. All transaction fees are charged from the bank account. The basic constraint on the consumption control $C$ and the portfolio selection control, denoted $(M, N)$, is that the investor must be solvent at all times. More precisely, if $X(t)$ and $Y(t)$ represent the amount of investment in the bank account and the stock, respectively, at time $t$, then we require $(X(t), Y(t)) \in \mathbb{S}$ for all $t \geq 0$, where

$$
\mathbb{S} \doteq\left\{(x, y) \in \mathbb{R}^{2}: x+(1+\lambda) y \geq 0 \text { and } x+(1-\mu) y \geq 0\right\} .
$$

These solvency constraints ensure that at all times the investor has sufficient wealth to settle any obligations due to selling stock short (the first inequality) or borrowing from the bank account (the second inequality). The goal of the investor is to maximize the expected total discounted utility of consumption, $\mathbb{E} \int_{0}^{\infty} e^{-\beta t} f(C(t)) d t$, where $\beta \in(0, \infty)$ is the discount factor and the utility function $f:[0, \infty) \rightarrow[0, \infty)$ is a continuous function satisfying $f(0)=0$. The condition $f(0)=0$ can be relaxed if $f$ is nondecreasing and $f(0)>-\infty$ by replacing $f$ with $f-f(0)$.

In the absence of transaction costs, Merton proved in the classical paper [25] that when the utility function is $f(c)=c^{p} / p, p<1, p \neq 0$ or $f(c)=\log c$ (note that the latter utility function does not satisfy the conditions of the current paper) the investor's optimal policy is to keep a constant proportion of total wealth in the risky asset and to consume at a rate proportional to total wealth. (For a simple and self-contained treatment, see [6]). This "Merton line" target can always be achieved since transactions can be made continuously and instantaneously without affecting wealth. However, when transaction costs apply, such a policy results in immediate bankruptcy. Magill and Constantinides first conjectured in [24] that there must exist a "no-transaction region" taking the form of a wedge in the wealth space. When wealth is inside this region, consumption is the only control that can be exercised. Purchase or sale of stock occurs only when the wealth attempts to exit the no-transaction region. The formal arguments of [24] were put on a rigorous footing by Davis and Norman in [6] for the cases $f(c)=c^{p} / p$ and $f(c)=\log c$. In their work, under suitable conditions on model parameters, the free boundary problem associated with optimal 
consumption in the presence of proportional transaction costs is solved explicitly, and $C^{2}$ regularity of the value function is established. The authors show that the (optimal) no-transaction region is a wedge; in particular, the optimal policy is to exercise the minimal amount of trading necessary to keep wealth inside the no-transaction region. Inside the region, consumption occurs at a finite rate. In [29] Shreve and Soner consider the same problem as in [6] but with conditions on the model parameters that are weaker and much more explicit. Once more, regularity properties of the value function and the associated free boundary are proved. A more general utility function, which satisfies suitable smoothness, concavity and growth properties, was considered in [31]. Using viscosity solution methods, the authors sketch a proof for unique solvability of the associated HJB equation by the value function. A finite difference approximation scheme for approximating the value function is introduced; however, convergence of the proposed scheme for the portfolio selection problem is not proved. The authors do provide results from several numerical studies which identify near optimal control policies and the (numerical) free boundary.

In the current work, we do not impose any concavity, smoothness, or growth conditions on the utility function; the key condition (Condition 2.1) that we require is that the value function is finite and continuous. In particular, we do not claim or use the fact that the value function is the unique solution of the associated HJB equation. The main goal of the study is to obtain convergent numerical approximations for the value function. The basic approach, as in [19], is to introduce a Markov decision problem (MDP) for an approximating, finite state, discrete time, controlled Markov chain. The main result of the paper (Theorem 5.12) shows that the value function of the MDP converges to the value function of the original singular control problem as various parameters in the approximation approach their limits suitably. In section 6 we use the approximating MDP to obtain computational schemes for obtaining near optimal control policies. The key result of this section is Lemma 6.1, which allows us to characterize the value function and optimal control policies via the solution of suitable dynamic programming equations (see Theorem 6.2). Finally, in section 7 results from a numerical study using the algorithm of section 6 are described. In particular, Figure 2 shows the numerical no-transaction region and the associated (numerical) free boundary obtained by an implementation of the algorithm.

The only paper (to the best of our knowledge) that carries out a complete convergence analysis for a numerical scheme for a singular control problem is [20]. Although several ideas developed in [20] are crucial to the ideas in the current paper, there are key differences in the model that make our analysis substantially delicate. First, the above paper considers a queuing problem with "finite buffers" which essentially means that the state space and control space are bounded. In the current study we first have to suitably approximate the original unbounded model by one in which the consumption control and the state space are bounded. This two stage approximation procedure is carried out in Propositions 2.2 and 2.4. This is the only place where the assumption on the continuity of the value function (Condition 2.1) is used. Next, in contrast to [20], in addition to singular control terms, we also have an absolutely continuous control term (consumption control) that appears in a nonlinear fashion in the cost (reward) criterion through the utility function $f$. This requires us to introduce the relaxed formulation for the stochastic control problem in order to carry out the convergence analysis. Lemma 5.1 ensures that the relaxed formulation does not change the value function of the control problem. The next substantial difficulty in our analysis is the state constraint feature of the dynamics. Although in [20] also the state is constrained to be in a bounded polyhedral region, that can be easily handled 
by introducing the so-called "Skorohod map." In the current model the directions of control, which do not point inward into the state space (see Figure 1), do not allow for a similar reduction. However, one useful feature of the dynamics (see (1)) is that once the state of the system reaches the boundary of $\mathbb{S}$, the only admissible control corresponds to moving the state process instantaneously to the origin and keeping it there at all times. This observation allows us to convert an infinite horizon cost to an exit time criterion (see (2)-(4)). This reformulation makes some aspects of the convergence analysis simpler; however, the degeneracies in the state dynamics make the treatment of convergence properties of exit times quite subtle. To see the basic difficulty, consider the following simple example. Let $\xi_{n}$ be a sequence of positive reals such that $\xi_{n} \rightarrow 0$ as $n \rightarrow \infty$. Let $x_{n}$ be the solution of the ODE $\dot{x}=x$ with initial condition $\xi_{n}$ and $x$ be the solution of the same ODE with 0 initial condition. Clearly $x_{n} \rightarrow x$ uniformly on compacts; however, if $\tau_{n} \doteq \inf \left\{t \mid x_{n}(t)=0\right\}$ and $\tau \doteq \inf \{t \mid x(t)=0\}$, then clearly $\tau_{n} \nrightarrow \tau$. In other words, convergence of processes in general need not imply the convergence of the corresponding exit times. The issue is especially problematic when, as is the case for the controlled dynamics considered in this paper, the diffusion coefficients in the state dynamics are not uniformly nondegenerate. This is another key difference between the current model and the problem studied in [20].

One of the major obstacles in proving the convergence of the value function of a sequence of approximating discrete MDPs to the value of the original singular control problem is proving the tightness of the sequence of singular control terms in the Skorohod $D[0, \infty)$ space. A powerful technique for bypassing this tightness issue, based on suitable stretching of a time scale, was introduced in [20]. Although such time transformation ideas go back to the work of Meyer and Zheng [27] (see also Kurtz $[18]$ ), the papers $[20,21]$ were the first to use such ideas in stochastic control problems. A similar technique was also recently used in [3]. A key ingredient to this technique is the uniform moment estimate obtained in Lemma 4.4. In [20] such a moment estimate follows easily from the form of the cost function where a strictly positive proportional cost is incurred for exercising the singular control. In the current problem there is no direct contribution to the (cost) reward function from the singular control term and, as a result, the proof of this uniform estimate becomes more involved. Roughly speaking, the main idea of the proof is that a controller cannot make too much use of a singular control without pushing the process to the boundary of the domain.

The paper is organized as follows. In section 2 we give a precise formulation of the control problem of interest. We also present here two propositions (Propositions 2.2 and 2.4) which allow approximation of the original control problem by one with a bounded state space and bounded consumption actions. Section 3 introduces the discrete MDP that approximates the original singular control problem, and section 4 defines the continuous time interpolations and the time transformation that are key to the convergence analysis. In section 5 we present the main convergence result that establishes the convergence of the value function of the MDP to that of the original singular control problem. Section 6 is devoted to computational methods for the MDP. A key result here is Lemma 6.1 which allows, via Theorems 6.2 and 6.3 , iterative methods for computation of the value function and optimal control policies for the MDP. In problems with only absolutely continuous controls, estimates of the form in Lemma 6.1 are straightforward consequences of a contraction property that follows from the strictly positive discount factor in the cost (cf. Chapter 6 of [19]). However, for singular control problems, due to the instantaneous nature of the control, such contraction estimates are typically unavailable. Here, once again, we 
use the special feature of the dynamics, which says that excessive use of the singular control will rapidly bring the process to the boundary, in obtaining such an estimate. In section 7 we present results from a numerical study of the algorithm. Finally, the appendix contains proofs of the more technical results.

The following notation will be used in the paper. Given a Polish space $E, D_{E} \equiv$ $D([0, \infty): E)$ will denote the space of paths that are right-continuous with left limits (RCLL) on $[0, \infty)$ taking values in $E$, endowed with the usual Skorohod topology. For an RCLL path $\{\xi(t)\}$, the jump at $t>0$ will be denoted by $\delta \xi(t)$. As a convention we take $\delta \xi(0) \doteq \xi(0)$. For a sequence of random variables $\left\{\xi_{n}\right\}_{n \geq 0}$, we will use the notation $\delta \xi_{n}$ for the increment $\xi_{n+1}-\xi_{n}$. For a point $x \in \mathbb{R}^{k}$ and a set $G \in \mathbb{R}^{k}$, $\operatorname{dist}(x, G)$ will denote the distance of $x$ from $\bar{G}$. The Borel sigma field for a metric space $E$ will be denoted by $\mathcal{B}(E)$.

2. Optimal consumption and portfolio selection with transaction costs. We begin with a precise mathematical formulation of the optimal consumption and investment problem described in the previous section. Let $(\Omega, \mathcal{F}, \mathbb{P})$ be a probability space on which is given a filtration $\left\{\mathcal{F}_{t}\right\}_{t \geq 0}$ satisfying the usual hypothesis. Let $W$ be a real-valued $\left\{\mathcal{F}_{t}\right\}$-Brownian motion. We will denote the probability system $\left(\Omega, \mathcal{F}, \mathbb{P},\left\{\mathcal{F}_{t}\right\}, W\right)$ by $\Phi$. The Wiener process represents the source of uncertainty of the risky asset. The state process, which represents the wealth of the investor, is a controlled Markov process $Z \equiv(X, Y)$ given on the above probability system via the equations

$$
\begin{aligned}
& d X(t)=(r X(t)-C(t)) d t-(1+\lambda) d M(t)+(1-\mu) d N(t), \\
& d Y(t)=b Y(t) d t+\sigma Y(t) d W(t)+d M(t)-d N(t),
\end{aligned}
$$

with initial condition $X(0-)=x, Y(0-)=y$, where $z \doteq(x, y) \in \mathbb{S}$. Here $C$ is an $\left\{\mathcal{F}_{t}\right\}$-progressively measurable process such that for all $t \in[0, \infty), C(t) \geq 0$ a.s. and $\mathbb{E} \int_{0}^{t} e^{-r s} C(s) d s<\infty$. Also, $M$ and $N$ are $\left\{\mathcal{F}_{t}\right\}$-adapted, nondecreasing, RCLL processes satisfying $M(0) \geq 0$ and $N(0) \geq 0$ a.s. The processes $X$ and $Y$ represent the amounts invested in the bond and the stock, respectively; $M(t), N(t)$ denote the cumulative purchases and sales of stock, respectively, over $[0, t]$. The process $C$ represents the consumption of the investor. The processes $C, M$, and $N$ are the control processes. Since $M$ and $N$ are not required to be absolutely continuous (with respect to the Lebesgue measure), they are referred to as singular controls. Denote by $\mathcal{A}(\Phi, z) \equiv \mathcal{A}(z)$ the set of "admissible controls," i.e., all $U \equiv(C, M, N)$ of the form described above. Let $\partial \mathbb{S}$ denote the boundary of $\mathbb{S}$. From the dynamical description of $Z$ it follows that if $z \in \partial \mathbb{S}$, then the only control that keeps the investor solvent takes $Z$ to the origin instantly and keeps it there at all times (see Figure 1).

Recall the utility function $f$ in the introduction. Since $f(0)=0$, one can reformulate the state constraint control problem on an infinite time horizon described in the introduction to an exit time control problem, as follows. For $z \in \mathbb{S}$ and $U \in \mathcal{A}(z)$, let $\tau \equiv \tau(z, U)$ be defined as

$$
\tau \doteq \inf \left\{t \in[0, \infty): Z(t) \notin \mathbb{S}^{o}\right\}
$$

where $Z$ is the controlled process corresponding to initial condition $z$ and control $U$. Define the cost, $J(z, U)$, for using the control $U$ by

$$
J(z, U) \doteq \mathbb{E} \int_{[0, \tau)} e^{-\beta t} f(C(t)) d t .
$$




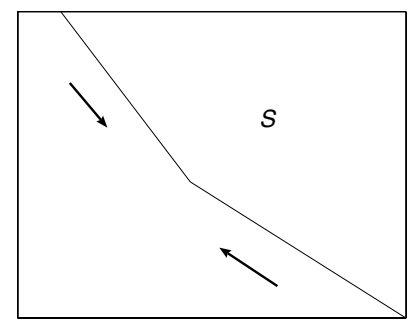

FIG. 1. State space and singular control directions.

The value function of the control problem is then given by

$$
V(z)=\sup _{\Phi} \sup _{U \in \mathcal{A}(z)} J(z, U)
$$

where the outside supremum is over all probability systems $\Phi$. The following will be a standing assumption in this work.

Condition 2.1. For all $z \in \mathbb{S}, V(z)<\infty$ and $V: \mathbb{S} \rightarrow \mathbb{R}_{+}$is a continuous map.

We refer the reader to $[16,29,31]$ for some sufficient conditions needed for the above assumption to hold.

State and control space truncation. In order to develop numerical methods for computing $V(z)$, we will need to first approximate the control problem by an analogous control problem with a bounded state space and control set. We now present the convergence result, which says that the value function of the "truncated control problem" converges to $V$ as the truncation parameters approach their limits. We begin by considering the control space truncation.

For $p \in(0, \infty)$, let $\mathcal{A}_{p}(\Phi, z) \equiv \mathcal{A}_{p}(z)$ be the subset of $\mathcal{A}(z)$ consisting of $U=$ $(C, M, N)$, which satisfy $0 \leq C(t) \leq p$, for all $t \geq 0$ a.s. Define $V_{p}(z)$ by replacing $\mathcal{A}(z)$ with $\mathcal{A}_{p}(z)$ in (4). The following is the first convergence result.

Proposition 2.2. $V_{p}$ converges to $V$, uniformly on compact subsets of $\mathbb{S}$, as $p \rightarrow \infty$.

Proof. We first establish pointwise convergence, i.e., $V_{p}(z) \rightarrow V(z)$ as $p \rightarrow \infty$. Since $V_{p}(z) \leq V(z)$, it suffices to show that, for all $z \in \mathbb{S}$,

$$
\liminf _{p \rightarrow \infty} V_{p}(z) \geq V(z)
$$

Fix $\epsilon>0$ and choose an " $\epsilon$-optimal control," i.e., $U_{\epsilon} \in \mathcal{A}(z)$ such that $V(z)-\epsilon<$ $J\left(z, U_{\epsilon}\right)$. Suppose $\tau_{\epsilon}$ is the associated exit time from $\mathbb{S}^{o}$. Define a control $\tilde{U}_{p} \equiv$ $\left(\tilde{C}_{p}, \tilde{M}_{p}, \tilde{N}_{p}\right)$ by $\tilde{C}_{p}(t) \doteq C_{\epsilon}(t) \wedge p, \tilde{M}_{p}(t) \doteq M_{\epsilon}(t), \tilde{N}_{p}(t) \doteq N_{\epsilon}(t), t \geq 0$. It follows from the fact that $\tilde{C}_{p} \leq C_{\epsilon}$ and standard comparison results for solutions of stochastic differential equations (cf. Proposition 5.2.18 of [17]) that the wealth process under control $\tilde{U}_{p}$ is never less than the wealth process under control $U_{\epsilon}$. In particular, denoting by $\tau_{p}$ the exit time from $\mathbb{S}^{o}$ by the controlled process corresponding to the control $\tilde{U}_{p}$, we have $\tau_{p} \geq \tau_{\epsilon}$. Combining this with the observations that $\tilde{C}_{p}(t) \uparrow C_{\epsilon}(t)$ as $p \rightarrow \infty$ a.s. for all $t \geq 0$ and $f$ is continuous, we have from Fatou's lemma

$$
\liminf _{p \rightarrow \infty} J\left(z, \tilde{U}_{p}\right) \geq \liminf _{p \rightarrow \infty} \mathbb{E} \int_{\left[0, \tau_{\epsilon}\right)} e^{-\beta t} f\left(\tilde{C}_{p}(t)\right) d t \geq \mathbb{E} \int_{\left[0, \tau_{\epsilon}\right)} e^{-\beta t} f\left(C_{\epsilon}(t)\right) d t \geq V(z)-\epsilon .
$$


Since $\epsilon>0$ is arbitrary, the pointwise convergence of $V_{p}$ to $V$ follows. Next we show that for each $p, V_{p}$ is lower semicontinuous (l.s.c.). Fix $z \in \mathbb{S}$ and let $\mathbb{S} \ni z_{n} \rightarrow z$ as $n \rightarrow \infty$. To prove that $V_{p}$ is l.s.c. it suffices to show that

$$
\liminf _{n \rightarrow \infty} V_{p}\left(z_{n}\right) \geq V_{p}(z) .
$$

Fix $\epsilon>0$ and let $U_{\epsilon}=\left(C_{\epsilon}, M_{\epsilon}, N_{\epsilon}\right) \in \mathcal{A}_{p}(z)$ be an $\epsilon$-optimal control, i.e., $V_{p}(z)-\epsilon<$ $J\left(z, U_{\epsilon}\right)$. Let $Z_{\epsilon}$ be the controlled process according to $U_{\epsilon}$ and define $\tau_{\epsilon}$ via (2) with $Z$ replaced by $Z_{\epsilon}$. Define $U_{n} \equiv\left(C_{n}, M_{n}, N_{n}\right)$ as $C_{n} \doteq C_{\epsilon}, M_{n}(t) \doteq M_{\epsilon}(t) 1_{t<\tau_{\epsilon}}+$ $M_{n}^{*} 1_{t \geq \tau_{\epsilon}}, N_{n}(t) \doteq N_{\epsilon}(t) 1_{t<\tau_{\epsilon}}+N_{n}^{*} 1_{t \geq \tau_{\epsilon}}$, where $M_{n}^{*}, N_{n}^{*} \geq 0$ are chosen so that the controlled process $Z_{n}$ corresponding to $U_{n}$ and initial condition $z_{n}$ satisfies $Z_{n}\left(\tau_{\epsilon}\right) \notin$ $\mathbb{S}^{o}$. (Note that, clearly, $U_{n} \in \mathcal{A}_{p}\left(z_{n}\right)$.) This ensures that $\tau_{n} \doteq \inf \left\{t: Z_{n}(t) \notin \mathbb{S}^{o}\right\}$ is at most $\tau_{\epsilon}$. Note that on the set $\left\{\tau_{\epsilon}=\infty\right\}$, we have $U_{n}(t)=U_{\epsilon}(t)$ for all $t \geq 0$. We claim that on the set $\left\{\tau_{\epsilon}<\infty\right\}$ we have $\lim _{n \rightarrow \infty} \tau_{n} \geq \tau_{\epsilon}$ a.s., which implies $\tau_{n} \rightarrow \tau_{\epsilon}$ a.s. as $n \rightarrow \infty$ on the set $\left\{\tau_{\epsilon}<\infty\right\}$. To see the claim, suppose that $\lim \inf \tau_{n}<\tau_{\epsilon}-\delta$ for some $\delta>0$. Then there exists $N_{0} \geq 1$ such that $\tau_{n}<\tau_{\epsilon}-\delta / 2$ for all $n \geq N_{0}$. Also, from the choice of the control $U_{n}$ we see that, for all $\delta>0$ and $L \in(0, \infty)$, $\sup _{0 \leq t \leq\left(\tau_{\epsilon}-\delta / 2\right) \wedge L}\left|Z_{n}(t)-Z(t)\right| \rightarrow 0$ in probability as $n \rightarrow \infty$. Combining this with the fact that $Z_{n}\left(\tau_{n}\right) \notin \mathbb{S}^{o}$, we have that $Z(t) \notin \mathbb{S}^{o}$ for some $t \leq \tau_{\epsilon}-\delta / 2$. However, this contradicts the definition of $\tau_{\epsilon}$. Thus we have shown $\tau_{n} \rightarrow \tau_{\epsilon}$ a.s. on the set $\left\{\tau_{\epsilon}<\infty\right\}$.

Next, recalling the choice of $U_{\epsilon}$ and that $C_{\epsilon}(t)=C_{n}(t)$ for all $t \geq 0$ on the set $\left\{\tau_{\epsilon}=\infty\right\}$, we have

$$
\begin{aligned}
V_{p}(z)-V_{p}\left(z_{n}\right) & \leq J\left(z, U_{\epsilon}\right)-J\left(z_{n}, U_{n}\right)+\epsilon \\
& =\mathbb{E}\left[1_{\left\{\tau_{\epsilon}<\infty\right\}} \int_{\left[\tau_{n}, \tau_{\epsilon}\right)} e^{-\beta t}\left(f\left(C_{\epsilon}(t)\right)-f\left(C_{n}(t)\right)\right) d t\right]+\epsilon \\
& \leq f_{*}(p) \mathbb{E}\left[1_{\left\{\tau_{\epsilon}<\infty\right\}} \int_{\left[\tau_{n}, \tau_{\epsilon}\right)} e^{-\beta t} d t\right]+\epsilon,
\end{aligned}
$$

where $f_{*}(p) \doteq \sup _{0 \leq c \leq p} f(c)<\infty$. Since $\tau_{n} \rightarrow \tau_{\epsilon}$ a.s. on the set $\left\{\tau_{\epsilon}<\infty\right\}$, the first term on the right in the last line above approaches 0 as $n \rightarrow \infty$. Inequality (5) now follows from the above display on taking $n \rightarrow \infty$ and then $\epsilon \rightarrow 0$. Finally, note that for each $z, V(z)-V_{p}(z) \downarrow 0$. The result now follows from Dini's theorem (cf. Theorem M8 in [1]).

Next, we consider the truncation of the state space. The reduction will be achieved by replacing the original dynamical system given by (1) with one which evolves exactly as before in the interior of some compact domain but is instantaneously reflected back when the controlled process is about to exit the domain. The reflection mechanism is made precise via the notion of a Skorohod map. We begin with the following definition. Fix $\ell \in(0, \infty)$.

Definition 2.3. Let $\phi \in \mathcal{D} \doteq D\left([0, \infty): \mathbb{R}^{2}\right)$ be such that $\phi(0) \in(-\infty, \ell] \times$ $(-\infty, \ell]$. We will denote the space of all such $\phi$ by $\mathcal{D}_{0}$. We say a pair $(\psi, \eta) \in \mathcal{D} \times \mathcal{D}$ solves the Skorohod problem $(S P)$ for $\phi$ in $(-\infty, \ell] \times(-\infty, \ell]$, with normal reflection, if the following hold:

(i) $\psi(0)=\phi(0)$.

(ii) $\psi(t)=\phi(t)-\eta(t), t \in(0, \infty)$.

(iii) $\psi(t) \in(-\infty, \ell] \times(-\infty, \ell]$ for all $t \geq 0$.

(iv) $\eta(\cdot)$ is componentwise nondecreasing. 
(v) $\eta_{i}(t)=\int_{(0, t]} 1_{\left\{\psi_{i}(t)=\ell\right\}} d \eta_{i}(t), i=1,2$, where $\eta(t)=\left(\eta_{1}(t), \eta_{2}(t)\right)^{\prime}, \psi(t)=$ $\left(\psi_{1}(t), \psi_{2}(t)\right)^{\prime}$.

It is well known (cf. $[7,13])$ that for every $\phi \in \mathcal{D}_{0}$, there is a unique solution $(\psi, \eta)$ to the above SP. We will write $\psi=\Gamma(\phi)$ and refer to the map $\Gamma: \mathcal{D}_{0} \rightarrow \mathcal{D}_{0}$ as the Skorohod map. The following Lipschitz property (cf. [7]) is quite useful in various estimates. There exists $\kappa \in(0, \infty)$, independent of $\ell$, such that, for all $\phi_{1}, \phi_{2} \in \mathcal{D}_{0}$,

$$
\left|\Gamma\left(\phi_{1}\right)-\Gamma\left(\phi_{2}\right)\right|_{T}^{*} \leq \kappa\left|\phi_{1}-\phi_{2}\right|_{T}^{*}, T \in(0, \infty) .
$$

We will now introduce the modified constrained dynamics of the controlled Markov process. Set $\mathbb{S}_{\ell} \doteq \mathbb{S} \cap(-\infty, \ell] \times(-\infty, \ell]$. Let $Z_{\ell} \equiv\left(X_{\ell}, Y_{\ell}\right)$ solve the following system of equations:

$$
\begin{aligned}
d X_{\ell}(t) & =\left(r X_{\ell}(t)-C(t)\right) d t-(1+\lambda) d M(t)+(1-\mu) d N(t)-d R_{1}(t), \\
d Y_{\ell}(t) & =b Y_{\ell}(t) d t+\sigma Y_{\ell}(t) d W(t)+d M(t)-d N(t)-d R_{2}(t),
\end{aligned}
$$

where $Z_{\ell}(0-)=z, U \equiv(C, M, N) \in \mathcal{A}_{p}(z), z=(x, y) \in \mathbb{S}_{\ell}$, and $R=\left(R_{1}, R_{2}\right)^{\prime}$ is a componentwise nondecreasing, RCLL, $\left\{\mathcal{F}_{t}\right\}$-adapted process satisfying

$$
{ }_{0}^{\infty} 1_{\left\{X_{\ell}(t)<\ell\right\}} d R_{1}(t)=0, \quad{ }_{0}^{\infty} 1_{\left\{Y_{\ell}(t)<\ell\right\}} d R_{2}(t)=0 .
$$

The unique solvability of (7) and (8) follows from the Lipschitz continuity property (6) of the Skorohod map and the usual Picard iteration method. Define $\tau_{\ell}$ and $J_{\ell}(z, U)$ as in (2) and (3) with $Z$ replaced by $Z_{\ell}$ in (2) and $\tau$ replaced by $\tau_{\ell}$ in (3). Define $V_{\ell, p}$ as

$$
V_{\ell, p}(z)=\sup _{\Phi} \sup _{U \in \mathcal{A}_{p}(\Phi, z)} J_{\ell}(z, U) .
$$

The following is the second convergence result of this section.

Proposition 2.4. For all $p \in(0, \infty), V_{\ell, p}$ converges to $V_{p}$, uniformly on compact subsets of $\mathbb{S}$, as $\ell \rightarrow \infty$.

Proof. Let $Z \equiv(X, Y)$ be as in (1) and $\tau$ as in (2), with $C \equiv 0$. It is easy to check that for each $T \in(0, \infty)$ and compact subset $\mathbb{S}_{0} \subset \mathbb{S}$, there exists $\Lambda \equiv \Lambda(T) \in(0, \infty)$ such that

$$
\sup _{\Phi} \sup _{(M, N)} \sup _{z \in \mathbb{S}_{0}} \mathbb{E} \sup _{0 \leq t \leq T \wedge \tau}\left(X^{+}(t)+Y^{+}(t)\right) \leq \Lambda,
$$

where the supremum is taken over all $\{\mathcal{F}(t)\}$-adapted, nondecreasing, RCLL processes $M$ and $N$ such that $M(0) \geq 0, N(0) \geq 0$, and over all systems $\Phi$. Thus in particular we have that

$$
\sup _{\ell} \sup _{\Phi} \sup _{U \in \mathcal{A}_{p}(\Phi, z)} \sup _{z \in \mathbb{S}_{0}} \mathbb{E} \sup _{0 \leq t \leq T \wedge \tau_{\ell}}\left(X_{\ell}^{+}(t)+Y_{\ell}^{+}(t)\right) \leq \Lambda,
$$

where $Z_{\ell} \equiv\left(X_{\ell}, Y_{\ell}\right)$ are as defined in (7), and $\tau_{\ell}$ is as introduced below (8).

Fix $\delta>0$. Let $z \in \mathbb{S}_{0}$ and $\epsilon>0$ be arbitrary. Let $\Phi$ and $U \in \mathcal{A}_{p}(z, \Phi)$ be such that $V_{\ell, p}(z) \leq J_{\ell}(z, U)+\epsilon$. Choose $T \in(0, \infty)$ such that $f_{*}(p) e^{-\beta T} / T<\epsilon$. Then $V_{\ell, p}(z) \leq \mathbb{E} \int_{0}^{T \wedge \tau_{\ell}} e^{-\beta t} f(C(t)) d t+2 \epsilon$.

Choose $\ell_{0} \equiv \ell_{0}(\delta)$ such that $\ell_{0}>\left(\Lambda f_{*}(p)\right) /(\delta \beta)$. Define

$$
A_{\ell_{0}} \doteq\left\{\omega: \sup _{0 \leq t \leq T \wedge \tau_{\ell}}\left(X_{\ell}^{+}(t)+Y_{\ell}^{+}(t)\right)>\ell_{0}\right\} \text {. }
$$


Then

$$
\begin{aligned}
& \mathbb{E}{ }_{0}^{T \wedge \tau_{\ell}} e^{-\beta t} f(C(t)) d t
\end{aligned}
$$

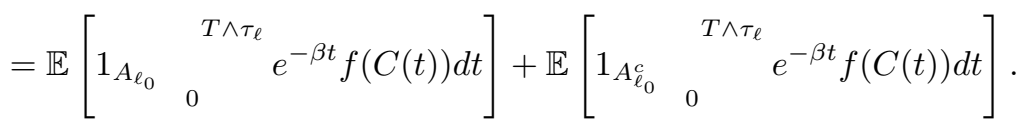

It follows from Markov's inequality and (10) that $\mathbb{P}\left[A_{\ell_{0}}\right] \leq \Lambda / \ell_{0}$. Thus the first integral on the right side of $(11)$ is bounded by $\left(f_{*}(p) / \beta\right) \mathbb{P}\left[A_{\ell_{0}}\right] \leq \delta$. Next, for $\ell \geq \ell_{0}$, on the set $A_{\ell_{0}}^{c}, Z_{\ell}\left(\cdot \wedge T \wedge \tau_{\ell}\right)=Z\left(\cdot \wedge T \wedge \tau_{\ell}\right)$. In particular, $T \wedge \tau \geq T \wedge \tau_{\ell}$. Thus

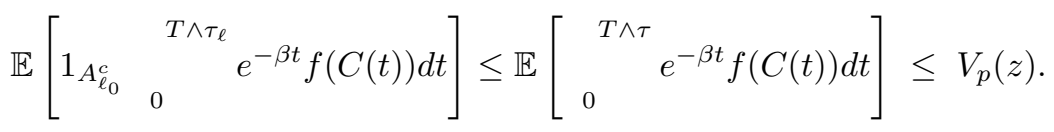

Combining the above bounds, we have $V_{\ell, p}(z) \leq V_{p}(z)+\delta+2 \epsilon$. Since $\epsilon>0$ is arbitrary, we have that, for all $\ell \geq \ell_{0}$ and $z \in \mathbb{S}_{0}, V_{\ell, p}(z) \leq V_{p}(z)+\delta$. It is easily seen that the roles of $V_{p}$ and $V_{\ell, p}$ can be interchanged in the above argument. Thus we have that, given $\delta>0$, there exists an $\ell_{0}$ such that $\left|V_{\ell, p}(z)-V_{p}(z)\right| \leq \delta$ if $\ell>\ell_{0}$, for all $z \in \mathbb{S}_{0}$. Since $\mathbb{S}_{0}$ is an arbitrary compact subset of $\mathbb{S}$, the result follows.

Corollary 2.5. For all $z \in \mathbb{S}, \lim _{p \rightarrow \infty} \lim _{\ell \rightarrow \infty} V_{\ell, p}(z)=V(z)$.

3. An approximating Markov decision problem. In this section we will present the Markov decision problem whose value function approximates $V_{\ell, p}$. Since throughout this section $\ell, p$ will be fixed, we will drop them from the notation: $V_{\ell, p}, \tau_{\ell}, J_{\ell}, \mathcal{A}_{p}(z)$ and $Z_{\ell} \equiv\left(X_{\ell}, Y_{\ell}\right)$. We will introduce a discrete time, discrete state controlled Markov chain to approximate the continuous time process given by (7).

Fix $h>0$ and define the two-dimensional $h$-grid, $L^{h} \doteq\{(j h, k h):-\infty<j, k<$ $+\infty\}$. The symbol $h$ denotes the approximation parameter, and as $h$ approaches 0 , a suitable interpolation of the controlled Markov chain, to be introduced below, "approaches" a controlled diffusion process of the form in (7). We will assume for simplicity that $\ell$ is an integer multiple of $h$.

A natural definition of the state space for the approximating chain is $\mathbb{S}_{\ell}^{h} \doteq \mathbb{S}_{\ell} \cap$ $L^{h}$. However, due to reflection terms in the dynamics of the controlled process, it is convenient to consider a slightly "enlarged" state space, namely, $\mathbb{S}_{\ell}^{h+} \doteq \mathbb{S}_{\ell+h} \cap$ $L^{h}$. The "solvency boundary" and reflecting boundary of the space $\mathbb{S}_{\ell}^{h+}$ are defined, respectively, as

$$
\begin{aligned}
& \partial^{h} \doteq\left\{(x, y) \in \mathbb{S}_{\ell}^{h+}: x+(1+\lambda) y \leq h(1+\lambda), \text { or } x+(1-\mu) y \leq h\right\} \\
& \partial_{\mathrm{R}}^{h} \doteq\left\{(x, y) \in \mathbb{S}_{\ell}^{h+}: x=\ell+h, \text { or } y=\ell+h\right\} .
\end{aligned}
$$

Let $\left\{Z_{n}^{h}, n=0,1,2, \ldots\right\}$ be a discrete time controlled Markov chain with state space $\mathbb{S}_{\ell}^{h+}$, with $Z_{n}^{h}=\left(X_{n}^{h}, Y_{n}^{h}\right)$. The transition probabilities will be defined so that the chain's evolution law well approximates the local behavior of the controlled diffusion (7). For each $n$, the increments of the chain $\delta Z_{n}^{h}$ will approximate exactly one of the following dynamical descriptions:

- "Controlled diffusion step": $\left(r X_{t}-C_{t}, b Y_{t}\right)^{\prime} d t+(0, \sigma)^{\prime} d W_{t}$.

- "Purchase control step": $(-(1+\lambda), 1)^{\prime} d M_{t}$.

- "Sales control step": $(1-\mu,-1)^{\prime} d N_{t}$.

- "Reflection step": $d R_{t}$. 
Each of these steps is described precisely in what follows. We also introduce a family of "interpolation intervals" $\left\{\Delta^{h}, h>0\right\}$ used in defining the approximating cost function and in the convergence arguments. For each pair $(z, c) \in \mathbb{S}_{\ell}^{h+} \times[0, p]$ we first define a family $\tilde{\Delta}^{h}(z, c)$. For the controlled diffusion steps, if the state of the chain is $z$ and the exercised consumption control is $c, \Delta^{h}$ will be taken to be $\tilde{\Delta}^{h}(z, c)$; whereas for singular control steps and reflection steps, $\Delta^{h}$ will be taken to be 0 . This reflects the fact that for the controlled diffusion (7), reflection and singular control terms can change the state instantaneously. Suitable conditions on $\tilde{\Delta}^{h}(z, c)$ needed to obtain convergence of the continuous time interpolated processes to corresponding controlled diffusions are introduced below.

Controlled diffusion steps and local consistency. By a controlled diffusion step we mean that the Markov chain evolves according to a transition law which is "locally consistent" in the sense of [19], with a (controlled) diffusion given as

$$
d \tilde{X}(t)=(r \tilde{X}(t)-C(t)) d t, d \tilde{Y}(t)=b \tilde{Y}(t) d t+\sigma \tilde{Y}(t) d W(t) .
$$

Formally, given $h>0$, we choose for each $c \in[0, p]$ and $z \in \mathbb{S}_{\ell}^{h+} \backslash \partial^{h}$ a probability measure $q_{h}^{(0)}(z, c, d \tilde{z})$ on $L^{h}$, along with an interpolation interval $\tilde{\Delta}^{h}(z, c)$, which satisfies the following local consistency conditions for some $\rho>0$ :

$$
\begin{aligned}
m_{0}(z, c) & \doteq \underset{L^{h}}{ }(\tilde{z}-z) q_{h}^{(0)}(z, c, d \tilde{z})=\left(\begin{array}{c}
r x-c \\
b y
\end{array}\right) \tilde{\Delta}^{h}(z, c)+O\left(h^{\rho} \tilde{\Delta}^{h}(z, c)\right), \\
\sigma_{0}(z, c) & \doteq \underset{L^{h}}{ }\left(\tilde{z}-z-m_{0}(z, c)\right)\left(\tilde{z}-z-m_{0}(z, c)\right)^{\prime} q_{h}^{(0)}(z, c, d \tilde{z}) \\
& =\left(\begin{array}{cc}
0 & 0 \\
0 & |\sigma y|^{2}
\end{array}\right) \tilde{\Delta}^{h}(z, c)+O\left(h^{\rho} \tilde{\Delta}^{h}(z, c)\right) .
\end{aligned}
$$

In the above displays, $\tilde{z}=(\tilde{x}, \tilde{y})$, and throughout, by the symbol $O(k)$ we will mean an expression which is bounded above by $\alpha|k|$, where $\alpha$ is a constant depending only on the coefficients of the model and the truncation parameters $\ell, p$. In addition we assume that there exists $\zeta \in(0, \infty)$ such that $q_{h}^{(0)}\left(z, c, B_{\zeta h}(z)\right)=1$ for all $c \in[0, p]$ and $h>0$, where $B_{\zeta h}(z)$ is a ball of radius $\zeta h$ centered at $z$. The interpolation intervals are required to satisfy

$$
\tilde{\Delta}_{*}^{h} \doteq \sup _{z, c} \tilde{\Delta}^{h}(z, c) \rightarrow 0 \text { as } h \rightarrow 0, \inf _{z, c} \tilde{\Delta}^{h}(z, c)>0 \text { for each } h>0,
$$

where the sup and inf in the above displays are taken over all $(z, c) \in \mathbb{S}_{\ell}^{h+} \times[0, p]$. For the sake of specificity we make the following choice for $q_{h}^{(0)}$. Let $Q(x, y) \equiv Q^{h}(x, y) \doteq$ $h r|x|+h p+h b|y|+\sigma^{2} y^{2}$. Define for all $(x, y) \in \mathbb{S}_{\ell}^{h+} \backslash \partial^{h}$

$$
\begin{aligned}
q_{h}^{(0)}((x, y), c,(x+h, y)) \doteq \frac{h r x^{+}}{Q(x, y)}, & q_{h}^{(0)}((x, y), c,(x-h, y)) \doteq \frac{h r x^{-}+h c}{Q(x, y)} \\
q_{h}^{(0)}((x, y), c,(x, y+h)) \doteq \frac{h b y^{+}+\frac{1}{2} \sigma^{2} y^{2}}{Q(x, y)}, & q_{h}^{(0)}((x, y), c,(x, y-h)) \doteq \frac{h b y^{-}+\frac{1}{2} \sigma^{2} y^{2}}{Q(x, y)} \\
q_{h}^{(0)}((x, y), c,(x, y)) & \doteq \frac{h(p-c)}{Q(x, y)} \\
\tilde{\Delta}^{h}(z, c) & \doteq \frac{h^{2}}{Q(x, y)} .
\end{aligned}
$$


It is easy to check that $q_{h}^{(0)}, \tilde{\Delta}^{h}$ defined above satisfy (12), (13), and (14).

Singular control steps. The singular control terms in the controlled diffusion are the nondecreasing RCLL processes $M$ and $N$. The process $M$ pushes the state process in the direction $v_{1}=(-(1+\lambda), 1)^{\prime}$, whereas $N$ pushes the state process in the direction $v_{2}=((1-\mu),-1)^{\prime}$. For the approximating chain we will assume that at most one among the sales control and purchase control are exercised at any given time instant and that the magnitude of the corresponding displacement is $O(h)$. In order to capture the "singular" behavior of the limit diffusion - namely, the feature that the state process can instantaneously be displaced by large amounts - we will take the interpolation interval for all singular control steps in the approximating chain to be 0 .

In order to obtain weak convergence of the interpolated chain to the controlled diffusion, we need to ensure that the control directions match asymptotically those for the physical problem. More precisely, given $h>0$ we define for each $z \in \mathbb{S}_{\ell}^{h+}$ two probability measures $q_{h}^{(i)}(z, d \tilde{z}), i=1,2$, on $L^{h}$ as follows. For states $(x, y) \in \mathbb{S}_{\ell}^{h+} \backslash \partial^{h}$,

$$
\begin{gathered}
q_{h}^{(1)}((x, y),(x-h, y))=\lambda /(\lambda+1), q_{h}^{(1)}((x, y),(x-h, y+h))=1 /(\lambda+1) \\
q_{h}^{(2)}((x, y),(x, y-h))=\mu, q_{h}^{(2)}((x, y),(x+h, y-h))=1-\mu .
\end{gathered}
$$

It is easy to check that $q_{h}^{(1)}$ and $q_{h}^{(2)}$ introduced above satisfy the following consistency conditions:

$$
\begin{aligned}
m_{i}(z) & \doteq{ }_{L^{h}}(\tilde{z}-z) q_{h}^{(i)}(z, d \tilde{z})=h v_{i}, \\
\sigma_{i}(z) & \doteq{ }_{L^{h}}\left(\tilde{z}-z-m_{i}(z)\right)\left(\tilde{z}-z-m_{i}(z)\right)^{\prime} q_{h}^{(i)}(z, d \tilde{z})=O\left(h^{2}\right) .
\end{aligned}
$$

Reflection steps. We will define a transition kernel that with probability 1 moves a state in $\partial_{\mathrm{R}}^{h}$ to some state in $\mathbb{S}_{\ell}^{h}$. Once more, since reflection in the diffusion control problem occurs instantaneously, we take the interpolation interval at reflection steps to be 0 . Since the directions of reflection in the diffusion control problem are normal, a natural choice of the transition kernel for reflection step is as follows for $z \in \partial_{\mathrm{R}}^{h}$ :

$$
q_{h}^{(3)}((\ell+h, y),(\ell, y))=1, \quad q_{h}^{(3)}((x, \ell+h),(x, \ell))=1, \quad q_{h}^{(3)}((\ell+h, \ell+h),(\ell, \ell))=1 .
$$

For $z \notin \partial_{\mathrm{R}}^{h}, q_{h}^{(3)}(z, \cdot)$ can be defined arbitrarily. It will be seen from the definition of admissible controls given below that for such states, the definition of $q_{h}^{(3)}$ is immaterial.

The controlled Markov chain. As described above, the control at each step is first specified by the choice of an action: controlled diffusion, singular control, or reflection. Therefore, we define a sequence of control actions $\left\{I_{n}^{h}, n=0,1,2, \ldots\right\}$ with $I_{n}^{h}=0,1,2,3$ if the $n$th step in the chain is a controlled diffusion step, purchase control step, sales control step, or reflection step, respectively. In the case of a controlled diffusion step, the magnitude of the consumption control must also be specified. Consequently, the space of controls is given by $\mathcal{U} \doteq\{0,1,2,3\} \times[0, p]$.

The probability measures associated with each of the control actions will now be combined into a single probability measure for use in defining the controlled Markov chain. For each $z \in \mathbb{S}_{\ell}^{h+} \backslash \partial^{h}, u \in \mathcal{U}(u=(i, c))$, we define a probability measure 
$p_{h}(z, u, d \tilde{z})$ on $L^{h}$ by

$$
p_{h}(z, u, d \tilde{z})=q_{h}^{(0)}(z, c, d \tilde{z}) 1_{\{i=0\}}+q_{h}^{(i)}(z, d \tilde{z}) 1_{\{i \in\{1,2,3\}\}} .
$$

The definition of the transition function for $z \in \partial^{h}$ is not important since in the analysis of the control problem the chain will be stopped the first time it hits $\partial^{h}$. For the sake of specificity we set $p_{h}(z, u, z)=1$ for all $z \in \partial^{h}$ and $u \in \mathcal{U}$.

We are now ready to specify the controlled Markov chains. Given a sequence $U^{h}=\left\{U_{n}^{h}, n=0,1,2, \ldots\right\}$ (where $U_{n}^{h}=\left(I_{n}^{h}, C_{n}^{h}\right)$ ) of $\mathcal{U}$-valued random variables we construct a controlled Markov chain $\left\{Z_{n}^{h}, n=0,1,2, \ldots\right\}$ with initial condition $z_{h}=\left(x_{h}, y_{h}\right) \in \mathbb{S}_{\ell}^{h+}$ and state space $\mathbb{S}_{\ell}^{h+}$, as follows:

$$
Z_{0}^{h}=z_{h}, \quad \mathbb{P}\left[Z_{n+1}^{h} \in E \mid \mathcal{F}_{n}^{h}\right]=p_{h}\left(Z_{n}^{h}, U_{n}^{h}, E\right), \quad n \geq 0, \quad E \in \mathcal{B}\left(\mathbb{S}_{\ell}^{h+}\right),
$$

where $\mathcal{F}_{n}^{h}=\sigma\left\{Z_{0}^{h}, \ldots, Z_{n}^{h}, U_{0}^{h}, \ldots, U_{n}^{h}\right\}$. The following definition of admissible controls ensures that $Z_{n}^{h} \in \mathbb{S}_{\ell}^{h+}$ for all $n$, and so the definition in (22) is meaningful.

Definition 3.1. The control sequence $U^{h}=\left\{U_{n}^{h}, n=0,1,2, \ldots\right\}$ is said to be admissible for the initial condition $z_{h}$, and $\left\{Z_{n}^{h}\right\}\left(\left\{Z_{n}^{h}, U_{n}^{h}\right\}\right)$ is called the corresponding controlled Markov chain (respectively, controlled pair) if

1. $U_{n}^{h}$ is $\sigma\left\{Z_{0}^{h}, \ldots, Z_{n}^{h}, U_{0}^{h}, \ldots, U_{n-1}^{h}\right\}$-adapted.

2. $\mathbb{P}\left[I_{n}^{h}=3 \mid Z_{n}^{h} \in \mathbb{S}_{\ell}^{h}\right]=0$ and $\mathbb{P}\left[I_{n}^{h}=3 \mid Z_{n}^{h} \in \partial_{R}^{h} \backslash \partial^{h}\right]=1$ for all $n$.

3. Condition (22) holds.

The class of all admissible control sequences for initial state $z_{h}$ will be denoted by $\mathcal{A}^{h}\left(z_{h}\right)$.

We also define for each $z \in \mathbb{S}_{\ell}^{h+}$ and $u=(i, c) \in \mathcal{U}$ the interpolation intervals

$$
\Delta^{h}(z, u)=\tilde{\Delta}^{h}(z, c) 1_{\{i=0\}} .
$$

For an admissible pair $\left\{Z_{n}^{h}, U_{n}^{h}\right\}$, we denote the associated sequence of interpolation intervals $\Delta^{h}\left(Z_{n}^{h}, U_{n}^{h}\right)$ by $\left\{\Delta_{n}^{h}, n=0,1,2, \ldots\right\}$. Define, $t_{0}^{h} \doteq 0$ and $t_{n}^{h} \doteq \sum_{i=0}^{n-1} \Delta_{i}^{h}$ for $n \geq 1$.

MDP for the chain. Given an admissible pair $\left\{Z_{n}^{h}, U_{n}^{h}\right\}$ let $\eta_{h} \doteq \inf \left\{n: Z_{n}^{h} \in\right.$ $\left.\partial^{h}\right\}$. The cost function for the controlled Markov chain is defined as

$$
J^{h}\left(z_{h}, U^{h}\right)=\mathbb{E} \sum_{n=0}^{\eta_{h}-1} e^{-\beta t_{n}^{h}} f\left(C_{n}^{h}\right)\left(\frac{1-e^{-\beta \Delta_{n}^{h}}}{\beta}\right) .
$$

Note that we have used the factor $\left(1-e^{-\beta \Delta_{n}^{h}}\right) / \beta$ rather than the more intuitive (and asymptotically equivalent) $\Delta_{n}^{h}$. This somewhat simplifies the convergence proofs without affecting the limiting results. The value function of the MDP is defined as

$$
V^{h}\left(z_{h}\right)=\sup _{U^{h} \in \mathcal{A}^{h}\left(z_{h}\right)} J^{h}\left(z_{h}, U^{h}\right) .
$$

4. Continuous time interpolation and time rescaling. One of the main goals of the study is to show that the value function of the MDP defined in (25) converges, as $h \rightarrow 0$, to the value function of the limit diffusion control problem. This convergence result allows for the computation of near optimal policies for the diffusion control problem introduced below (6) by numerically solving the above MDP. We next introduce the continuous time interpolation and time rescaling techniques that will be used in the proof of our main convergence result. 
The continuous time interpolations of various processes will be constructed to be piecewise constant on the time intervals $\left[t_{n}^{h}, t_{n+1}^{h}\right), n \geq 0$. For use in this construction we define $n^{h}(t) \doteq \max \left\{n: t_{n}^{h} \leq t\right\}, t \geq 0$. Note that $n^{h}(t)$ is an $\left\{\mathcal{F}_{n}^{h}\right\}$-stopping time. Setting $\mathcal{F}^{h}(t) \doteq \mathcal{F}_{n^{h}(t)}^{h}$ we obtain a continuous time filtration $\left\{\mathcal{F}^{h}(t), t \geq 0\right\}$. Define $U^{h}(t) \doteq U_{n^{h}(t)}^{h}, t \geq 0$. Also, define the continuous time processes associated with the controlled diffusion steps as follows. First, let $B_{0}^{h}=0$ and $S_{0}^{h}=0$ and define for $n \geq 1$,

$$
B_{n}^{h} \doteq{ }_{k=0}^{n \wedge \eta_{h}-1} \mathbb{E}\left[\delta Z_{k}^{h} \mid \mathcal{F}_{k}^{h}\right] 1_{\left\{I_{k}^{h}=0\right\}}, \quad S_{n}^{h} \doteq{ }_{k=0}^{n \wedge \eta_{h}-1}\left(\delta Z_{k}^{h}-\mathbb{E}\left[\delta Z_{k}^{h} \mid \mathcal{F}_{k}^{h}\right]\right) 1_{\left\{I_{k}^{h}=0\right\}} .
$$

Define the continuous time process $B^{h}$ by setting $B^{h}(0) \doteq 0$ and $B^{h}(t) \doteq B_{n^{h}(t)}^{h}$ for $t>0$. The process $S^{h}$ is defined in a similar manner. We define the interpolations associated with the purchase control and sales control as follows. Let $M_{0}^{h}=0, N_{0}^{h}=0$, $E_{i, 0}^{h}=0, i=1,2$, and define for $n \geq 1$,

$$
M_{n}^{h} \doteq{ }_{k=0}^{n \wedge \eta_{h}-1} h 1_{\left\{I_{k}^{h}=1\right\}}, \quad N_{n}^{h} \doteq{ }_{k=0}^{n \wedge \eta_{h}-1} h 1_{\left\{I_{k}^{h}=2\right\}}, \quad E_{i, n}^{h} \doteq{ }_{k=0}^{n \wedge \eta_{h}-1}\left(\delta Z_{k}^{h}-h v_{i}\right) 1_{\left\{I_{k}^{h}=i\right\}} .
$$

The continuous time processes $M^{h}$ and $N^{h}$ are defined as $M^{h}(0) \doteq 0, N^{h}(0) \doteq 0$ and $M^{h}(t) \doteq M_{n^{h}(t)}^{h}, N^{h}(t) \doteq N_{n^{h}(t)}^{h}$ for $t \geq 0$. The processes $E_{1}^{h}$ and $E_{2}^{h}$ are defined analogously. The continuous time process associated with reflection is defined as follows. If $n^{h}(t)=0$, define $R^{h}(t)=0$; otherwise let

$$
R^{h}(t) \doteq-{ }_{k=0}^{n^{h}(t)-1} \delta Z_{k}^{h} 1_{\left\{I_{k}^{h}=3\right\}}
$$

We define the continuous time interpolation $Z^{h}$ of the controlled Markov chain $Z_{n}^{h}$ introduced in Definition 3.1 by $Z^{h}(t) \doteq Z_{n^{h}(t)}^{h}, t \geq 0$. The following representation for $Z^{h}(t)$ is easily verified:

$$
Z^{h}(t)=z_{h}+B^{h}(t)+S^{h}(t)+v_{1} M^{h}(t)+v_{2} N^{h}(t)+E_{1}^{h}(t)+E_{2}^{h}(t)-R^{h}(t), t \geq 0 .
$$

Also, it follows from condition (12) that on the set $\left\{I_{n}^{h}=0, \eta_{h}>n\right\}$,

$$
\mathbb{E}\left[\delta Z_{n}^{h} \mid \mathcal{F}_{n}^{h}\right]=\left(\begin{array}{c}
r X_{n}^{h}-C_{n}^{h} \\
b Y_{n}^{h}
\end{array}\right) \Delta^{h}\left(Z_{n}^{h}, 0, C_{n}^{h}\right)+O\left(h^{\rho} \Delta^{h}\left(Z_{n}^{h}, 0, C_{n}^{h}\right)\right) \text { a.s. }
$$

This fact, together with the piecewise constant nature of the processes, yields

$$
B^{h}(t)={ }_{0}^{t \wedge \tau^{h}}\left(\begin{array}{c}
r X^{h}(s)-C^{h}(s) \\
b Y^{h}(s)
\end{array}\right) d s+\delta_{1}^{h}(t),
$$

where $\tau^{h} \doteq t_{\eta_{h}}^{h}$ and $\delta_{1}^{h}$ is an $\left\{\mathcal{F}^{h}(t)\right\}$-adapted process which, in view of (14), satisfies for all $t \geq 0$ and $m \geq 1$,

$$
\sup _{0 \leq s \leq t} \mathbb{E}\left|\delta_{1}^{h}(s)\right|^{m} \rightarrow 0 \text { as } h \rightarrow 0
$$


A similar calculation gives the following representation of the cost function (24):

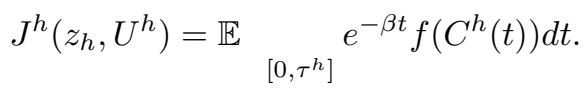

Time rescaling. A common approach for proving the convergence of $V^{h}$ to $V$ as $h \rightarrow 0$ is to begin by showing that the collection $\left\{\left(Z^{h}(\cdot), \tau^{h}\right), h \geq 0\right\}$ is tight and then characterize the subsequential weak limits suitably. However, for problems with singular controls, showing the tightness of the above family becomes problematic since, in general, the processes $\left\{\left(M^{h}(\cdot), N^{h}(\cdot)\right), h \geq 0\right\}$ may fail to be tight. A powerful method for handling this tightness issue was introduced by Martins and Kushner [21]. The basic idea is to suitably stretch out the time scale so that the various processes involved in the convergence analysis, in the new time scale, are tight; carry out the weak convergence analysis with the rescaled processes; and finally, revert back to the original time scale to argue the convergence of $V^{h}$ to $V$.

We now introduce the time rescaling that will be used in our study. The rescaled time increments, $\left\{\hat{\Delta}_{n}^{h}, n=0,1,2, \ldots\right\}$, are defined as $\hat{\Delta}_{n}^{h} \doteq \Delta_{n}^{h} 1_{\left\{I_{n}^{h}=0\right\}}+h 1_{\left\{I_{n}^{h} \in\{1,2\}\right\}}$. Define $\hat{t}_{0}^{h} \doteq 0$ and $\hat{t}_{n}^{h} \doteq{ }_{i=0}^{n-1} \hat{\Delta}_{i}^{h}$ for $n \geq 1$.

DEFINITION 4.1. The rescaled time process $\hat{T}^{h}(t)$ is the unique continuous nondecreasing process satisfying the following: (1) $\hat{T}^{h}(0)=0$; (2) the derivative of $\hat{T}^{h}(t)$ is 1 for $t \in\left(\hat{t}_{n}^{h}, \hat{t}_{n+1}^{h}\right)$ if $I_{n}^{h}=0$; (3) the derivative of $\hat{T}^{h}(t)$ for $t \in\left(\hat{t}_{n}^{h}, \hat{t}_{n+1}^{h}\right)$ is 0 if $I_{n}^{h}=1,2,3$.

It is easy to check that $\hat{T}^{h}\left(\hat{t}_{n}^{h}\right)=t_{n}^{h}$ and that $\hat{T}^{h}\left(\hat{t}_{n+1}^{h}\right)-\hat{T}^{h}\left(\hat{t}_{n}^{h}\right)=\Delta_{n}^{h}$. Let $\hat{n}^{h}(t) \doteq \max \left\{n: \hat{t}_{n}^{h} \leq t\right\}, t \geq 0$. Using the observation that every reflection step must be followed by either a singular control step or a diffusion control step, it follows that $\hat{n}^{h}(t)$ is a bounded $\left\{\mathcal{F}_{n}^{h}\right\}$-stopping time, with bound

$$
\hat{n}^{h}(t) \leq 2 \quad \frac{t}{h}+\frac{t}{\inf _{z, c} \hat{\Delta}^{h}(z, 0, c)}<\infty .
$$

Define the continuous time filtration $\left\{\hat{\mathcal{F}}^{h}(t), t \geq 0\right\}$ by setting $\hat{\mathcal{F}}^{h}(t) \doteq \mathcal{F}_{\hat{n}^{h}(t)}$.

The rescaled processes (denoted with $\mathrm{a}^{\wedge}$ ) are defined in a manner similar to the processes defined below (26) with appropriate adjustments to the time variable. For example, we define $\hat{B}^{h}(0)=0$ and $\hat{B}^{h}(t) \doteq B_{\hat{n}^{h}(t)}^{h}$ if $\hat{n}^{h}(t)>0$. We define the processes $\hat{U}^{h}(t), \hat{S}^{h}(t), \hat{M}^{h}(t), \hat{N}^{h}(t), \hat{E}_{1}^{h}(t), \hat{E}_{2}^{h}, \hat{R}^{h}(t), \hat{Z}^{h}(t)$ analogously (that is, by replacing $n^{h}(t)$ with $\hat{n}^{h}(t)$ in the definitions below (26)). Then we have the following rescaled version of $(28)$ :

$$
\hat{Z}^{h}(t)=z_{h}+\hat{B}^{h}(t)+\hat{S}^{h}(t)+v_{1} \hat{M}^{h}(t)+v_{2} \hat{N}^{h}(t)+\hat{E}_{1}^{h}(t)+\hat{E}_{2}^{h}-\hat{R}^{h}(t) .
$$

Remark 4.2. From the definition of $\hat{T}^{h}(t)$ if follows that $\hat{n}^{h}(t)=n^{h}\left(\hat{T}^{h}(t)\right)$. This equality yields a straightforward relationship between the original interpolated processes and the rescaled processes. For example, $\hat{B}^{h}(t)=B^{h}\left(\hat{T}^{h}(t)\right)$. Similar equations hold between $U^{h}(t), S^{h}(t), M^{h}(t), N^{h}(t), E_{1}^{h}(t), E_{2}^{h}, R^{h}(t), Z^{h}(t)$, and their corresponding rescaled versions.

Using the fact that $\hat{T}^{h}\left(\hat{t}_{n+1}^{h}\right)-\hat{T}^{h}\left(\hat{t}_{n}^{h}\right)=\Delta_{n}^{h}$, which is 0 for singular control and reflection steps, a calculation similar to that which produced (29) yields

$$
\hat{B}^{h}(t)={ }_{0}^{t \wedge \hat{\tau}_{1}^{h}}\left(\begin{array}{c}
r \hat{X}^{h}(s)-\hat{C}^{h}(s) \\
b \hat{Y}^{h}(s)
\end{array}\right) d \hat{T}^{h}(s)+\hat{\delta}_{1}^{h}(t),
$$


where $\hat{\tau}_{1}^{h} \doteq \inf \left\{t: \hat{Z}^{h}(t) \in \partial^{h}\right\}$ and $\hat{\delta}_{1}^{h}$ is an $\left\{\hat{\mathcal{F}}^{h}(t)\right\}$-adapted process satisfying, for all $m \geq 1$,

$$
\mathbb{E} \sup _{0 \leq s \leq t}\left|\hat{\delta}_{1}^{h}(s)\right|^{m} \rightarrow 0 \text { as } h \rightarrow 0 .
$$

We now state several lemmas related to the time rescaling. The following "change of variables" formula (cf. Theorem IV.3.45 in [28]) will be used several times in our analysis.

LEMma 4.3. Let $\hat{G}:[0, \infty) \rightarrow[0, \infty)$ be a continuous and nondecreasing function. Suppose that $\hat{G}(t) \rightarrow \infty$ as $t \rightarrow \infty$. Define the inverse $G:[0, \infty) \rightarrow[0, \infty)$ as $G(t)=$ $\inf \{s: \hat{G}(s)>t\}$. Then for all bounded and measurable functions $g:[0, \infty) \rightarrow[0, \infty)$,

$$
{ }_{[0, G(t)]} g(s) d \hat{G}(s)={ }_{[0, t]} g(G(s)) d s .
$$

The following lemma is at the heart of the time transformation idea. It ensures that the weak limits of $\hat{T}^{h}(t)$ increase to $\infty$ as $t \rightarrow \infty$ and thus makes reverting back to the original time scale, in the limit, possible (see Theorem 5.6). The proof of the lemma is contained in the appendix.

Lemma 4.4. Let $\left\{U_{n}^{h}, n=0,1,2, \ldots\right\}_{h>0}$ be a family of admissible control sequences. Then for all $t \geq 0$,

$$
\sup _{h} \mathbb{E}\left|M^{h}(t)+N^{h}(t)\right|<\infty .
$$

An important consequence of the above lemma is the following.

Lemma 4.5. There exists an $h_{0} \in(0, \infty)$ such that for all $h<h_{0}, \hat{T}^{h}(t) \rightarrow \infty$ with probability 1 as $t \rightarrow \infty$.

Proof. Since $\tilde{\Delta}_{*}^{h} \rightarrow 0$ as $h \rightarrow 0$, we can find an $h_{0}$ such that $\tilde{\Delta}_{*}^{h}<1$ for all $h<h_{0}$. We will argue via contradiction. Suppose $h<h_{0}$ and $\mathbb{P}\left[\sup _{t \geq 0} \hat{T}^{h}(t)<\infty\right]>0$. Then there exist $\epsilon>0$ and $T_{0}>0$ such that

$$
\mathbb{P}\left[\sup _{t \geq 0} \hat{T}^{h}(t)<T_{0}-1\right]>\epsilon .
$$

Using Lemma 4.4 we can find a $K$ large enough so that

$$
\mathbb{P}\left[M^{h}\left(T_{0}\right) \geq K\right] \leq \frac{\mathbb{E} M^{h}\left(T_{0}\right)}{K}<\frac{\epsilon}{4}, \quad \mathbb{P}\left[N^{h}\left(T_{0}\right) \geq K\right] \leq \frac{\mathbb{E} N^{h}\left(T_{0}\right)}{K}<\frac{\epsilon}{4} .
$$

We will now show that

$$
\mathbb{P}\left[\hat{T}^{h}\left(T_{0}+2 K\right)<T_{0}-1\right] \leq \frac{\epsilon}{2} .
$$

This will lead to a contradiction in view of (37) and hence prove the lemma. Note that

$$
\begin{aligned}
& \mathbb{P}\left[\hat{T}^{h}\left(T_{0}+2 K\right)<T_{0}-1\right] \\
& \quad \leq \mathbb{P}\left[\hat{T}^{h}\left(T_{0}+M^{h}\left(T_{0}\right)+N^{h}\left(T_{0}\right)\right)<T_{0}-1, M^{h}\left(T_{0}\right)<K, N^{h}\left(T_{0}\right)<K\right] \\
& \quad+\mathbb{P}\left[M^{h}\left(T_{0}\right) \geq K\right]+\mathbb{P}\left[N^{h}\left(T_{0}\right) \geq K\right] \\
& \quad \leq \mathbb{P}\left[\hat{T}^{h}\left(T_{0}+M^{h}\left(T_{0}\right)+N^{h}\left(T_{0}\right)\right)<T_{0}-1\right]+\frac{\epsilon}{4}+\frac{\epsilon}{4} .
\end{aligned}
$$


Furthermore, for each fixed $t, t+M^{h}(t)+N^{h}(t) \geq{ }_{k=0}^{n^{h}(t)-1}\left(\Delta_{k}^{h} 1_{\left\{I_{k}^{h}=0\right\}}+h 1_{\left\{I_{k}^{h}=1,2\right\}}\right)$. Since $\hat{T}^{h}$ is nondecreasing and $\hat{T}^{h}\left(\hat{t}_{n}^{h}\right)=t_{n}^{h}$,

$$
\begin{aligned}
\hat{T}^{h}\left(t+M^{h}(t)+N^{h}(t)\right) & \geq \hat{T}^{h}\left({ }_{k=0}^{n^{h}(t)-1}\left(\Delta_{k}^{h} 1_{\left\{I_{k}^{h}=0\right\}}+h 1_{\left\{I_{k}^{h}=1,2\right\}}\right)\right) \\
& =\hat{T}^{h}\left(\hat{t}_{n^{h}(t)}^{h}\right)=t_{n^{h}(t)}^{h}={ }_{k=0}^{n^{h}(t)-1} \Delta_{k}^{h} 1_{\left\{I_{k}^{h}=0\right\}} \geq t-\tilde{\Delta}_{*}^{h} .
\end{aligned}
$$

The last inequality above is a consequence of the inequalities $\begin{aligned} & n^{h}(t)-1 \\ & k=0\end{aligned} \Delta_{k}^{h} 1_{\left\{I_{k}^{h}=0\right\}} \leq$ $t \leq{ }_{k=0}^{n^{h}(t)} \Delta_{k}^{h} 1_{\left\{I_{k}^{h}=0\right\}}$. Recalling that $\tilde{\Delta}_{*}^{h}<1$, we see that $\hat{T}^{h}\left(t+M^{h}(t)+N^{h}(t)\right) \geq$ $t-1$ for all $t \geq 0$. Using this inequality in (39) proves (38) and hence we have the result.

Let $T^{h}(t) \doteq \inf \left\{s: \hat{T}^{h}(s)>t\right\}$. Observe that $\hat{T}^{h}\left(T^{h}(t)\right)=t$ and that, due to Lemma 4.5, $T^{h}(t)<\infty$ a.s. for all $t \geq 0$. Define $\hat{\tau}^{h} \doteq T^{h}\left(\tau^{h}\right)$.

Lemma 4.6. For $z_{h} \in \mathbb{S}_{\ell}^{h+}$ and $\left\{U_{n}^{h}\right\} \in \mathcal{A}^{h}\left(z_{h}\right)$,

$$
J^{h}\left(z_{h}, U^{h}\right)=\mathbb{E} \underset{\left[0, \hat{\tau}_{1}^{h}\right]}{ } e^{-\beta \hat{T}^{h}(t)} f\left(\hat{C}^{h}(t)\right) d \hat{T}^{h}(t) .
$$

Proof. Note that

$$
\hat{\tau}_{1}^{h}=\inf \left\{t: \hat{Z}^{h}(t) \in \partial^{h}\right\}=\inf \left\{t: Z^{h}\left(\hat{T}^{h}(t)\right) \in \partial^{h}\right\}=\inf \left\{t: \hat{T}^{h}(t) \geq \tau^{h}\right\} .
$$

If $\tau^{h}=\infty$, then clearly $\hat{\tau}_{1}^{h}=\infty$. Suppose $\tau^{h}<\infty$. Then the above display shows that $\hat{\tau}_{1}^{h}=T^{h}\left(\tau^{h}-\right)$. Also, clearly $\hat{T}^{h}$ is constant over the interval $\left(T^{h}\left(\tau^{h}-\right), T^{h}\left(\tau^{h}\right)\right]$. The result now follows from (30) and Lemma 4.3.

5. Main convergence result. In this section we show that $V^{h}\left(z_{h}\right)$ converges to $V(z)$ whenever $z_{h} \rightarrow z$. The basic approach will be as follows. First, we establish tightness of the continuous time (rescaled) processes defined in the previous section and characterize their subsequential limits. Then we define a time transformation for the limit processes to revert back to the original scale. We will show that the time transformed versions of the limit processes have the same laws as those of the various processes in the diffusion control problem. Using this characterization result we will show that, given a sequence of admissible controls $\left\{U^{h}, h>0\right\}$, the limsup of the corresponding cost functions is bounded above by the cost for an admissible control for the diffusion control problem. This will establish that $\limsup _{h \rightarrow 0} V^{h}\left(z_{h}\right) \leq V(z)$ whenever $z_{h} \rightarrow z$. Finally, we prove convergence of the value functions by proving the reverse inequality. The main idea of this proof is to select a near optimal control for the limit diffusion control problem and to construct from this an admissible control for the controlled Markov chain which is asymptotically near optimal.

We begin by introducing the following "relaxed control" formulation which arises naturally in the weak convergence arguments for convergence of the cost functions.

Relaxed control formulation. Let $\tilde{\mathcal{M}}$ denote the space of all Borel measures $\vartheta$ on $[0, p] \times[0, \infty)$ such that if $\vartheta(d \alpha, d t)=\vartheta_{t}(d \alpha) \nu(d t)$, then (i) $\vartheta_{t}$ is a probability measure on $[0, p]$ for $\nu$-almost every $t$, and (ii) $\nu(a, b] \leq b-a$ for all $0 \leq a \leq b<\infty$. Let $\mathcal{M}$ be the subset of $\tilde{\mathcal{M}}$ consisting of $\vartheta$ that satisfy, for all $t \geq 0, \vartheta([0, p] \times[0, t])=t$. Given a probability system $\Phi$ and initial condition $z \in \mathbb{S}_{\ell}$, let $\overline{\mathcal{A}}_{p}(\Phi, z)$ be the set of 
all processes $\bar{U} \equiv(m, M, N)$, where $M$ and $N$ are as introduced below (1), $m \in \mathcal{M}$ a.s., and $m(A \times[0, t])$ is $\left\{\mathcal{F}_{t}\right\}$-adapted for all $t \in[0, \infty), A \in \mathcal{B}[0, p]$. Set $C(t) \doteq$ $\int_{[0, p]} \alpha m_{t}(d \alpha)$, where $m_{t}$, a probability measure on $[0, p]$, is defined by the relation $m(d \alpha, d t)=m_{t}(d \alpha) d t$.

Let $Z$ be defined via (7) with $(C, M, N)$ as above and $\tau$ be given by (2). Define for $\bar{U} \in \overline{\mathcal{A}}_{p}(\Phi, z)$,

$$
\bar{J}(z, \bar{U}) \doteq \mathbb{E} \underset{[0, p] \times[0, \tau)}{ } e^{-\beta t} f(\alpha) m(d \alpha, d t)
$$

and let

$$
\bar{V}(z) \doteq \sup _{\Phi} \sup _{\bar{U} \in \overline{\mathcal{A}}(\Phi, z)} \bar{J}(z, \bar{U}) .
$$

The following lemma establishes the equivalence between the relaxed control formulation and the precise control formulation. The proof is contained in the appendix.

Lemma 5.1. For all $z \in \mathbb{S}, \bar{V}(z)=V(z)$.

The space $\tilde{\mathcal{M}}$ can be metrized using the Prohorov metric in the usual way (see pages 263-264 of [19]). Furthermore, with this metric, $\tilde{\mathcal{M}}$ is a compact space, and a sequence $\vartheta_{n} \in \tilde{\mathcal{M}}$ converges to $\vartheta$ if and only if for all continuous functions $\psi$ on $[0, p] \times[0, \infty)$ with compact support,

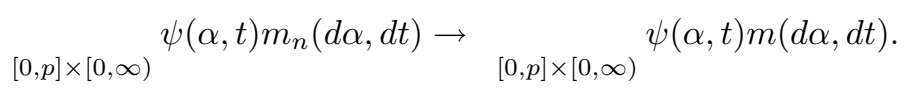

We now define $\tilde{\mathcal{M}}$-valued random variables $\hat{m}^{h}$ by the relation

$$
\hat{m}^{h}(A \times[0, t]) \doteq \underset{[0, t]}{1} 1_{A}\left(\hat{C}^{h}(s)\right) d \hat{T}^{h}(s), \quad A \in \mathcal{B}([0, p]), t \in[0, \infty) .
$$

Noting that the right side above is equal to $\int_{[0, t]}\left(\int_{A} \delta_{\hat{C}^{h}(s)}(d \alpha)\right) d \hat{T}^{h}(s)$, where $\delta_{x}$ is the probability measure concentrated at $x$, we can write $\hat{m}^{h}(d \alpha, d t)$ as $\hat{m}_{t}^{h}(d \alpha) \hat{\nu}^{h}(d t)$, where $\hat{m}_{t}^{h}$ and $\hat{\nu}^{h}$ are given by $\hat{m}_{t}^{h}(A)=\delta_{\hat{C}^{h}(t)}(A), \hat{\nu}^{h}(a, b]=\hat{T}^{h}(b)-\hat{T}^{h}(a)$ for $A \in \mathcal{B}([0, p])$ and $0 \leq a \leq b<\infty$.

Convergence of the time rescaled processes. Recall the definitions and notation, found in section 4, relating to the continuous time interpolated processes and the corresponding rescaled versions. We begin by showing that the processes $\hat{E}_{1}^{h}$ and $\hat{E}_{2}^{h}$ converge weakly to the 0 process as $h \rightarrow 0$.

Lemma 5.2. Let $\hat{E}_{i}^{h}, i=1,2$, be as defined above (27). Then $\hat{E}_{i}^{h}$ converges in probability to 0 in $D\left([0, \infty): \mathbb{R}^{2}\right)$.

Proof. The local consistency condition (18) and property (22) imply that $E_{i, n}^{h}$ is an $\left\{\mathcal{F}_{n}^{h}\right\}$-martingale. As $\hat{n}^{h}(t)$ is a bounded stopping time (cf. (31)) and the increments of $E_{i, n}^{h}$ are bounded, it follows from the optional sampling theorem that the continuous time process $\hat{E}_{i}^{h}(t)$ is an $\left\{\hat{\mathcal{F}}^{h}(t)\right\}$-martingale, the trace of the quadratic variation of which is given by $\operatorname{Tr}\left\langle\hat{E}_{i}^{h}\right\rangle(t)={ }_{k=0}^{\hat{n}^{h}(t) \wedge \eta_{h}-1} \mathbb{E}\left[\left|\delta z_{k}^{h}-h v_{i}\right|^{2} 1_{\left\{I_{k}^{h}=i\right\}} \mid \mathcal{F}_{k-1}^{h}\right]$. Finally, applying Doob's inequality, (19), and the observation that the maximum number of steps of either singular control in the first $\hat{n}^{h}(t)$ steps is $t / h$, we have for $i=1,2, \mathbb{E}\left[\sup _{s \leq t}\left|\hat{E}_{i}^{h}(s)\right|\right]^{2} \leq 4 \mathbb{E} \operatorname{Tr}\left\langle\hat{E}_{1}^{h}\right\rangle(t) \leq O\left(h^{2}\right)(t / h)=O(h)$. The result now follows. 
Define the process $\hat{A}^{h}$ by $\hat{A}^{h}(t) \doteq \int_{[0, t)} \hat{C}^{h}(s) d \hat{T}^{h}(s)$. Let $\overline{\mathbb{R}}$ denote the one point compactification of $\mathbb{R}$. The following proposition gives the tightness of the various time rescaled processes. The proof is similar to that of Theorem 5.3 of [20] and is therefore omitted.

Proposition 5.3. Let $\hat{H}^{h} \doteq\left(\hat{Z}^{h}, \hat{T}^{h}, \hat{A}^{h}, \hat{M}^{h}, \hat{N}^{h}, \hat{R}^{h}, \hat{B}^{h}, \hat{S}^{h}\right)$. Then the family $\left\{\left(\hat{H}^{h}, \hat{\tau}_{1}^{h}, \hat{m}^{h}\right), h>0\right\}$ is tight in $D([0, \infty): E) \times \overline{\mathbb{R}} \times \mathcal{M}$, where $E=\mathbb{S}_{\ell}^{h+} \times \mathbb{R}_{+}^{6} \times \mathbb{R}^{4}$.

We now turn our attention to characterizing subsequential limit points of the family $\left\{\left(\hat{H}^{h}, \hat{\tau}_{1}^{h}, \hat{m}^{h}\right), h>0\right\}$. Suppose that the initial condition sequence $\left\{z_{h}\right\}$ converges to some $z \in \mathbb{S}_{\ell}$. Slightly abusing notation, let $h$ index a weakly convergent subsequence of $\left(\hat{H}^{h}, \hat{\tau}_{1}^{h}, \hat{m}^{h}\right)$ with weak limit, $\left(\hat{H}, \hat{\tau}_{1}, \hat{m}\right)$, where $\hat{H} \doteq(\hat{Z}, \hat{T}, \hat{A}, \hat{M}, \hat{N}, \hat{R}, \hat{B}, \hat{S})$, given on some probability space $(\Omega, \mathcal{F}, \mathbb{P})$. Let $\hat{\mathcal{F}}^{*}(t) \doteq \sigma(\hat{H}(s), \hat{m}(A \times[0, s)) \mid A \in$ $\mathcal{B}([0, p]), 0 \leq s \leq t)$, and let $\hat{\mathcal{F}}(t) \doteq \hat{\mathcal{F}}^{*}(t+) \vee \mathcal{N}$, where $\mathcal{N}$ denotes the collection of all $\mathbb{P}$-null sets.

THEOREM 5.4. The limit point $\left(\hat{H}, \hat{\tau}_{1}, \hat{m}\right)$ has the following properties.

1. $\hat{T}$ is nondecreasing and Lipschitz continuous with Lipschitz coefficient 1.

2. There exists an $\{\hat{\mathcal{F}}(t)\}$-progressively measurable process $\hat{C}$ with $\hat{C}(t) \in[0, p]$ for all $t \geq 0$, such that

$$
\hat{B}(t)={ }_{0}^{t \wedge \hat{\tau}_{1}}\left(\begin{array}{c}
r \hat{X}(s)-\hat{C}(s) \\
b \hat{Y}(s)
\end{array}\right) d \hat{T}(s) .
$$

3. $\hat{S}_{1}(t)=0$, for all $t \geq 0$, and $\hat{S}_{2}$ is a continuous $\{\hat{\mathcal{F}}(t)\}$-martingale with quadratic variation $\left\langle\hat{S}_{2}\right\rangle_{t}=\int_{0}^{t}|\sigma \hat{Y}(s)|^{2} d \hat{T}(s), t \geq 0$.

4. $\hat{M}$ and $\hat{N}$ are nondecreasing and continuous.

5. $\hat{R}$ is a vector of nondecreasing continuous processes which satisfy

$$
{ }_{0}^{\infty} 1_{\{\hat{X}(t)<\ell\}} d \hat{R}_{1}(t)=0, \quad{ }_{0}^{\infty} 1_{\{\hat{Y}(t)<\ell\}} d \hat{R}_{2}(t)=0 .
$$

6. $\hat{Z}$ is a continuous process satisfying $\mathbb{P}\left[\hat{Z}(t) \in S_{\ell}\right]=1$ for all $t \geq 0$ and

$$
\hat{Z}(t)=z+\hat{B}(t)+\hat{S}(t)+v_{1} \hat{M}(t)+v_{2} \hat{N}(t)-\hat{R}(t) .
$$

7. Writing $\hat{m}(d \alpha, d t)$ as $\hat{m}_{t}(d \alpha) \hat{\nu}(d t)$ we have $\hat{\nu}(a, b]=\hat{T}(b)-\hat{T}(a), 0 \leq a \leq$ $b<\infty$.

8. $\hat{C}(t)=\int_{[0, p]} \alpha \hat{m}_{t}(d \alpha)$ for $\hat{\nu}$-almost every $t \in[0, \infty)$.

Proof. By appealing to the Skorohod representation theorem and by relabeling the convergent subsequence, we can assume without loss of generality that $\hat{H}^{h} \rightarrow \hat{H}$ a.s. The fact that the process $\hat{T}$ is nondecreasing and Lipschitz continuous with Lipschitz coefficient 1 follows easily from similar properties for $\hat{T}^{h}$. Since $\left|\hat{A}^{h}(t)-\hat{A}^{h}(s)\right| \leq$ $p\left|\hat{T}^{h}(t)-\hat{T}^{h}(s)\right|$ it follows that $\hat{A}$ is absolutely continuous with respect to $\hat{T}$. Therefore there exists a $[0, p]$-valued process $\hat{C}$, progressively measurable with respect to $\left\{\hat{\mathcal{F}}^{*}(t)\right\}$ such that $\hat{A}(t)=\int_{0}^{t} \hat{C}(s) d \hat{T}(s)$. This fact, together with $\left(\hat{Z}^{h}, \hat{T}^{h}\right) \rightarrow(\hat{Z}, \hat{T})$ a.s. and an application of the dominated convergence theorem, yields 2 . We next show that $\hat{S}$ has continuous paths. First, note that by local consistency $((12),(13))$ there exists $\zeta \in(0, \infty)$ such that for all $u \geq 0, h \geq 0 j\left(\hat{S}^{h}, u\right) \doteq \sup _{t \leq u}\left|\hat{S}^{h}(t)-\hat{S}^{h}(t-)\right| \leq 2 \zeta h$. Thus for $h$ small enough, $j\left(\hat{S}^{h}\right) \doteq \int_{0}^{\infty} e^{-u}\left(j\left(\hat{S}^{h}, u\right) \wedge 1\right) d u \leq 2 \zeta h$. Therefore, by Theorem 3.10.2 in [9] the limiting process $\hat{S}$ has continuous paths. One can check 
that the quadratic variation of $\hat{S}^{h}$, which is an $\left\{\hat{\mathcal{F}}^{h}(t)\right\}$-martingale, is given by

$$
\left\langle\hat{S}^{h}\right\rangle(t)=\left(\begin{array}{ll}
0 & 0 \\
0 & 1
\end{array}\right){ }_{0}^{t \wedge \hat{\tau}_{1}^{h}}\left|\sigma \hat{Y}^{h}(s)\right|^{2} d \hat{T}^{h}(s)+\hat{\delta}_{2}^{h}(t)
$$

where due to (14) and using (31) the process $\hat{\delta}_{2}^{h}$ satisfies for all $m \geq 1$,

$$
\mathbb{E} \sup _{s \leq t}\left|\hat{\delta}_{2}^{h}(s)\right|^{m} \rightarrow 0 \text { as } h \rightarrow 0 .
$$

From (44) it follows that $\hat{S}_{1}(t)=0$ for all $t \geq 0$. From (44) and the Burkholder-Gundy inequalities we also have

$$
\mathbb{E}\left|\hat{S}_{2}^{h}(t)\right|^{4} \leq \alpha \quad T^{2}+\mathbb{E} \sup _{0 \leq u \leq t}\left|\hat{\delta}_{2}^{h}(u)\right|^{2}
$$

Thus the family $\left\{\left(\hat{S}_{2}^{h}(t)\right)^{2}, h>0\right\}$ is uniformly integrable. A standard argument (cf. pages $1457-1458$ in [20]) shows that $\hat{S}_{2}$ is an $\left\{\hat{\mathcal{F}}_{t}\right\}$-martingale with quadratic variation as given in 3. Part 4 is immediate on noting that $\hat{M}^{h}, \hat{N}^{h}$ are nondecreasing, and since the maximum number of purchase or sales steps over $\left(\hat{n}^{h}(t), \hat{n}^{h}(t+s)\right)$ is $s / h+1$,

$$
\left|\hat{M}^{h}(t+s)-\hat{M}^{h}(t)\right| \leq s+h,\left|\hat{N}^{h}(t+s)-\hat{N}^{h}(t)\right| \leq s+h .
$$

From Definition 3.1(3) it follows that (42) holds with $(\hat{X}, \hat{Y}, \hat{R})$ replaced by $\left(\hat{X}^{h}\right.$, $\left.\hat{Y}^{h}, \hat{R}^{h}\right)$. Also clearly $\hat{Z}^{h} \in(-\infty, \ell] \times(-\infty, \ell]$. Parts 5 and 6 are now immediate consequences of (32) and continuity properties of the Skorohod map (see (6)). Next, write $\hat{m}(d \alpha, d t)$ as $\hat{m}_{t}(d \alpha) \hat{\nu}(d t)$. Since $\hat{m}^{h}([0, p],(a, b])=\hat{T}^{h}(b)-\hat{T}^{h}(a)$ for $0 \leq a \leq b<\infty$, taking limits yields $\hat{\nu}(a, b]=\hat{T}(b)-\hat{T}(a)$. This proves part 7. Part 8 is immediate from the representation $\int_{(a, b]} \hat{C}^{h}(s) d \hat{T}^{h}(s)=\int_{(a, b] \times[0, p]} \alpha \hat{m}^{h}(d \alpha, d s)$, $0 \leq a \leq b<\infty$ and the fact that (see the proof of part 2) $\int_{(a, b]} \hat{C}^{h}(s) d \hat{T}^{h}(s)$ converges to $\int_{(a, b]} \hat{C}(s) d \hat{T}(s)$.

Time inversion. We now define an inverse time transformation that will revert the limit processes back to the original time scale. We will see that the time inverted processes lead to an admissible control pair for the diffusion control problem in (7)-(9). The key step in returning to the original time scale is the following result analogous to Lemma 4.5.

LEMMA 5.5. $\hat{T}(t) \rightarrow \infty$ with probability 1 as $t \rightarrow \infty$.

Proof. We will argue via contradiction. Suppose $\mathbb{P}\left[\sup _{t \geq 0} \hat{T}(t)<\infty\right]>0$. Then there exist $\epsilon>0$ and $T_{0}>0$ such that

$$
\mathbb{P} \sup _{t \geq 0} \hat{T}(t)<T_{0}-1>\epsilon .
$$

As in the proof of Lemma 4.5 we can find a $K \in(0, \infty)$ such that $\lim _{\operatorname{lnf}} \rightarrow 0 \mathbb{P}\left[\hat{T}^{h}\left(T_{0}+\right.\right.$ $\left.2 K)<T_{0}-1\right] \leq \epsilon / 2$. The weak convergence $\hat{T}^{h} \Rightarrow \hat{T}$ now implies $\mathbb{P}\left[\hat{T}\left(T_{0}+2 K\right)<\right.$ $\left.T_{0}-1\right] \leq \epsilon / 2$. This contradicts (45), and hence the result follows.

Let $T$ be the inverse of $\hat{T}$, defined as $T(t) \doteq \inf \{s: \hat{T}(s)>t\}$. From Lemma 5.5 it follows that $T(t)<\infty$ a.s. for all $t \geq 0$. Since $\hat{T}(t)$ is nondecreasing and continuous, 
it follows that $T(t)$ is nondecreasing and right-continuous. Also note the following properties for all $t \geq 0$ :

$$
\begin{aligned}
T(t) \geq t, & \hat{T}(T(t))=t, \quad T(\hat{T}(t)) \geq t, \\
T(t) \uparrow \infty \text { as } t \uparrow \infty, & T(t)<\infty \text { a.s., } \quad \hat{T}(s) \in[0, t] \Leftrightarrow s \in[0, T(t)] .
\end{aligned}
$$

Let $\hat{H}$ be as in Theorem 5.4. Define $H(t) \doteq \hat{H}(T(t))$. We will use similar notation for the various components of $H$; for example, $Z(t) \doteq \hat{Z}(T(t))$, etc. Let $\tau_{1} \doteq \hat{T}\left(\hat{\tau}_{1}\right)$. Then by (43), for $t \geq 0$,

$$
Z(t)=z+B(t)+S(t)+v_{1} M(t)+v_{2} N(t)-R(t) .
$$

Before characterizing the various terms in (47) we note that for $t \geq 0,\{T(s)<$ $t\}=\{\hat{T}(t)>s\} \in \hat{\mathcal{F}}(t)$ since $\hat{T}(t)$ is $\hat{\mathcal{F}}(t)$-measurable. Therefore, since $\hat{\mathcal{F}}(t)$ is right-continuous, $T(s)$ is an $\{\hat{\mathcal{F}}(t)\}$-stopping time for each $s \geq 0$. Let $\mathcal{F}_{0}(t) \doteq \hat{\mathcal{F}}(T(t))$ and note that $H(t) \doteq \hat{H}(T(t))$ and $m(A \times[0, t]) \doteq \hat{m}(A \times[0, T(t)])$ are $\mathcal{F}_{0}(t)$ measurable. Define $\mathcal{F}(t) \doteq \sigma(H(s), m(A \times[0, s]): A \in \mathcal{B}([0, p]), 0 \leq s \leq t)$. Then $\mathcal{F}(t) \subseteq \mathcal{F}_{0}(t)$.

THEOREM 5.6. The processes in (47) have the following properties.

1. $B(t)=\int_{0}^{t \wedge \tau_{1}}\left(\begin{array}{c}r X(s)-C(s) \\ b Y(s)\end{array}\right) d s$.

2. $S_{1} \equiv 0$ and $S_{2}$ is a continuous $\left\{\mathcal{F}_{0}(t)\right\}$-martingale with quadratic variation

$$
\left\langle S_{2}\right\rangle(t)={ }_{0}^{t \wedge \tau_{1}}|\sigma Y(s)|^{2} d s
$$

There exists an enlargement of the probability space $(\Omega, \mathcal{F}, \mathbb{P})$ and of the filtration $\left\{\mathcal{F}_{0}(t)\right\}$ that supports a Wiener process $W$, which is a martingale with respect to the enlarged filtration and such that

$$
S_{2}(t)={ }_{0}^{t \wedge \tau_{1}} \sigma Y(s) d W(s)
$$

3. The process $C$ is $\left\{\mathcal{F}_{0}(t)\right\}$-progressively measurable with $C(t) \in[0, p]$ a.s. for all $t \geq 0$. In addition, $M(0), N(0) \geq 0$ and the processes $M$ and $N$ are right-continuous, nondecreasing, and $\left\{\mathcal{F}_{0}(t)\right\}$-adapted. Hence defining $\Phi \doteq$ $\left(\Omega, \mathcal{F}, \mathbb{P},\left\{\mathcal{F}_{0}(t)\right\}, W\right)$ we have $U \equiv(C, M, N) \in \mathcal{A}_{p}(\Phi, z)$.

4. For all $t \geq 0, Z(t) \in(-\infty, \ell] \times(-\infty, \ell]$ a.s., $R$ is a vector of nondecreasing right-continuous processes, and the representation (7) holds with $\left(X_{\ell}, Y_{\ell}, Z_{\ell}\right)$ there replaced by $(X, Y, Z)$.

Remark 5.7. Note that Theorem 5.6 does not prove that $(Z, R)$ is a solution to the Skorohod problem introduced in Definition 2.3, since in general (8) may fail to hold for the process $R$. However, note that if $\left(Z^{*}, R^{*}\right)$ is the solution of $(7)$ and (8) with $U=(C, M, N)$ as in part 3 of Theorem 5.6 , then by classical comparison results for (reflected) stochastic differential equations one can show that $Z^{*}(t) \geq Z(t)$ for all $t$ a.s., and so $\tau^{*} \geq \tau$ where $\tau$ is as in (2) and $\tau^{*}$ is defined by the right side of (2) with $Z$ replaced by $Z^{*}$. This in particular shows that

$$
{ }_{[0, p] \times[0, \tau]} e^{-\beta t} f(\alpha) d m_{t}(d \alpha) d t \leq{ }_{[0, p] \times\left[0, \tau^{*}\right]} e^{-\beta t} f(\alpha) d m_{t}(d \alpha) d t .
$$


Proof of Theorem 5.6. Part 1 is an immediate consequence of Theorem 5.4(2) and Lemma 4.3 on noting that

$$
1_{\left[T\left(\tau_{1}-\right), T\left(\tau_{1}\right)\right]} d \hat{T}(s)=0 \text { a.s. }
$$

Clearly, $S_{1}(t) \doteq \hat{S}_{1}(T(t)) \equiv 0$ a.s. From Theorem 5.4 we have that $\left\{\hat{S}_{2}(t), \hat{\mathcal{F}}(t)\right\}$ is a continuous martingale. Thus for all $n \geq 1, \mathbb{E}\left[\hat{S}_{2}(T(t) \wedge n) \mid \hat{\mathcal{F}}(T(s))\right]=\hat{S}_{2}(T(s) \wedge$ $n)$ a.s. Also as $\hat{S}_{2}$ has continuous paths and $T(t)<\infty$ a.s., we have as $n \rightarrow \infty$ for all $t \geq 0, \hat{S}_{2}(T(t) \wedge n) \rightarrow \hat{S}_{2}(T(t))=S_{2}(t)$ a.s. Furthermore, from Theorem 5.4, part 3, there exists $\alpha \in(0, \infty)$ such that $\mathbb{E}\left|\hat{S}_{2}(T(t) \wedge n)\right|^{2} \leq \alpha t$ for all $t \geq$ $0, n \in \mathbb{N}$. Hence, for each fixed $t$, the family $\left\{\hat{S}_{2}(T(t) \wedge n), n \geq 1\right\}$ is uniformly integrable and therefore $\hat{S}_{2}(T(t) \wedge n) \rightarrow \hat{S}_{2}(T(t))$ in $L^{1}$. Taking limits as $n \rightarrow \infty$, it follows that $\mathbb{E}\left[\hat{S}_{2}(T(t)) \mid \hat{\mathcal{F}}(T(s))\right]=\hat{S}_{2}(T(s))$, that is, $\mathbb{E}\left[S_{2}(t) \mid \mathcal{F}_{0}(s)\right]=S_{2}(s)$. This proves that $\left\{S_{2}(t), \mathcal{F}_{0}(t)\right\}$ is a martingale. Although $T$ in general may fail to be continuous, $S_{2}(\cdot) \doteq \hat{S}_{2}(T(\cdot))$ has continuous paths a.s. This is a consequence of the fact that $\left\{S_{2}^{h}(\cdot)\right\}_{h>0}$ is tight in $D([0, \infty))$, and an argument similar to that for $\left\{\hat{S}_{2}^{h}\right\}$ in the proof of Theorem 5.4 shows that any weak limit point, $\tilde{S}_{2}$, of $\left\{S_{2}^{h}\right\}$ must have continuous paths a.s. Also, since $\hat{S}_{2}^{h}(\cdot)=S_{2}^{h}\left(\hat{T}^{h}(\cdot)\right)$, we must have that if $\left(\tilde{S}_{2}, \hat{S}_{2}, \hat{T}\right)$ is a limit point of the tight sequence $\left(S_{2}^{h}, \hat{S}_{2}^{h}, \hat{T}^{h}\right)$, then $\hat{S}_{2}(t)=\tilde{S}_{2}(\hat{T}(t))$, and thus from (46), $S(t) \doteq \hat{S}_{2}(T(t))=\tilde{S}_{2}(t)$. Thus we have shown that $S_{2}$ is a continuous $\mathcal{F}_{0}(t)$ martingale. We next consider its quadratic variation. By the Burkholder-DavisGundy inequalities (cf. Theorem 3.3.28 in [17]) there exists a constant $\alpha$ independent of $n$ such that

$$
\mathbb{E}\left[\left|\hat{S}_{2}^{2}(T(t) \wedge n)\right|^{2}\right] \leq \mathbb{E}\left[\sup _{0 \leq s \leq T(t)}\left|\hat{S}_{2}(s \wedge n)\right|^{4}\right] \leq \alpha\left(\alpha_{1}^{2} t^{2}\right) .
$$

Thus the families $\left\{\hat{S}_{2}^{2}(T(t) \wedge n), n \geq 1\right\}$ and $\left\{\left\langle\hat{S}_{2}\right\rangle(T(t) \wedge n), n \geq 1\right\}$ are uniformly integrable for each fixed $t \geq 0$. Since $\hat{S}_{2}^{2}$ (respectively, $\left.\left\langle\hat{S}_{2}\right\rangle\right)$ has continuous paths and $T(t)<\infty$ a.s. $\hat{S}^{2}(T(t) \wedge n) \rightarrow \hat{S}_{2}^{2}(T(t))$ (respectively, $\left\langle\hat{S}_{2}\right\rangle(T(t) \wedge n) \rightarrow\left\langle\hat{S}_{2}\right\rangle(T(t))$ ) a.s. as $n \rightarrow \infty$. By the uniform integrability, this convergence also holds in the $L^{1}$ sense. Thus

$$
\mathbb{E}\left[\hat{S}_{2}^{2}(T(t) \wedge n)-\left\langle\hat{S}_{2}\right\rangle(T(t) \wedge n) \mid \hat{\mathcal{F}}(T(s))\right] \rightarrow \mathbb{E}\left[\hat{S}_{2}^{2}(T(t))-\left\langle\hat{S}_{2}\right\rangle(T(t)) \mid \hat{\mathcal{F}}(T(s))\right] .
$$

The above relation and the fact that $\hat{S}_{2}^{2}-\left\langle\hat{S}_{2}\right\rangle$ is an $\hat{\mathcal{F}}_{t}$-martingale now show that $\mathbb{E}\left[S_{2}^{2}(t)-\langle\hat{S}\rangle(T(t)) \mid \mathcal{F}_{0}(s)\right]=S_{2}^{2}(s)-\langle\hat{S}\rangle(T(s))$. Thus the quadratic variation of $S_{2}$ is given by $\left\langle S_{2}\right\rangle(t)=\left\langle\hat{S}_{2}\right\rangle(T(t))$. The representation (48) now follows on using Theorem 5.4, Lemma 4.3, and (51). By the martingale representation theorem (e.g., Theorem 3.4.2 in [17]) it now follows that there exists a one-dimensional Brownian motion $W$, possibly defined on an enlarged probability space, that is, a martingale with respect to an enlargement of the filtration $\left\{\mathcal{F}_{0}(t)\right\}$ and is such that (49) holds.

The $\left\{\mathcal{F}_{0}(t)\right\}$-progressive measurability (respectively, adaptedness) of $C$ (respectively, $M$ and $N$ ) follows from the $\{\hat{\mathcal{F}}(t)\}$-progressive measurability of $\hat{C}$ (respectively, adaptedness of $\hat{M}$ and $\hat{N}$ ). Also, since $\hat{C}$ takes values in $[0, p]$, the same is true of $C$. Right continuity of $M$ and $N$ is a consequence of the fact that $\hat{M}$ and $\hat{N}$ are continuous and $T$ is right continuous. This proves 3. Part 4 is once more an immediate consequence of Theorem 5.4 (part (5)) and Lemma 4.3. 
Until now the parameters $\ell, p$ have been fixed and thus excluded from the notation. It is convenient to include these parameters in the notation for the remainder of this section.

Convergence of the value functions. Let $z \in \mathbb{S}_{\ell}$ and let $\left\{z_{h}, h>0\right\}$ be a sequence with $z_{h} \in \mathbb{S}_{\ell}^{h}$ such that $z_{h} \rightarrow z$ as $h \rightarrow 0$. Recall the definitions of $V_{\ell, p}(z)$ in (9) and $V^{h}\left(z_{h}\right)$ in (25). Our main goal in this section is to show that $V^{h}\left(z_{h}\right) \rightarrow V_{\ell, p}(z)$ as $h \rightarrow 0$. We begin with the following proposition.

Proposition 5.8. Let $\left\{z_{h}\right\}, z$ be as above. Then $\limsup _{h \rightarrow 0} V^{h}\left(z_{h}\right) \leq V_{\ell, p}(z)$.

Proof. Fix for each $h>0$ an admissible control sequence for the initial condition $z_{h}, U^{h} \equiv\left\{U_{n}^{h}, n \geq 1\right\} \in \mathcal{A}^{h}\left(z_{h}\right)$. Recall the definition of $J^{h}\left(z_{h}, U^{h}\right)$ in (24). In order to prove the proposition it suffices to show that

$$
\limsup _{h \rightarrow 0} J^{h}\left(z_{h}, U^{h}\right) \leq V_{\ell, p}(z) .
$$

Using Lemma 4.6 and boundedness of $f$, we can find, for each $\epsilon \in(0, \infty)$, a $c \equiv c(\epsilon) \epsilon$ $(0, \infty)$ such that

$$
J^{h}\left(z_{h}, U^{h}\right) \leq \mathbb{E} \underset{\left[0, \hat{\tau}_{1}^{h} \wedge c\right] \times[0, p]}{ } e^{-\beta \hat{T}^{h}(t)} f(\alpha) \hat{m}^{h}(d \alpha, d t)+\frac{\epsilon}{2} .
$$

Let $\left(\hat{H}^{h}, \hat{\tau}_{1}^{h}, \hat{m}^{h}\right)$ be as in Proposition 5.3 and $\left(\hat{H}, \hat{\tau}_{1}, \hat{m}\right)$ be one of its weak limit points. Once more, as in the proof of Theorem 5.4 we can assume, by relabeling and appealing to the Skorohod representation theorem, that $\left(\hat{H}^{h}, \hat{\tau}_{1}^{h}, \hat{m}^{h}\right) \rightarrow\left(\hat{H}, \hat{\tau}_{1}, \hat{m}\right)$ a.s. Taking limits as $h \rightarrow 0$ in (54), we have

$$
\limsup _{h \rightarrow 0} J^{h}\left(z_{h}, U^{h}\right) \leq \mathbb{E} \underset{[0, p] \times\left[0, \hat{\tau}_{1} \wedge c\right]}{ } e^{-\beta \hat{T}(t)} f(\alpha) \hat{m}(d \alpha, d t)+\frac{\epsilon}{2} .
$$

As $\epsilon>0$ and $c=c(\epsilon)$ are arbitrary,

$$
\limsup _{h \rightarrow 0} J^{h}\left(z_{h}, U^{h}\right) \leq \mathbb{E} \underset{[0, p] \times\left[0, \hat{\tau}_{1}\right]}{ } e^{-\beta \hat{T}(t)} f(\alpha) \hat{m}(d \alpha, d t) .
$$

Let, as before, $\hat{\tau} \doteq \inf \left\{t \geq 0: \hat{Z}(t) \notin \mathbb{S}^{o}\right\}$. Recall that $\hat{\tau}_{1} \geq \hat{\tau}$. Then clearly

$$
\begin{aligned}
\mathbb{E} & 1_{\{\hat{\tau}=\infty\}} \underset{[0, p] \times\left[0, \hat{\tau}_{1}\right]}{ } e^{-\beta \hat{T}(t)} f(\alpha) \hat{m}(d \alpha, d t) \\
= & \mathbb{E} 1_{\{\hat{\tau}=\infty\}} \underset{[0, p] \times[0, \hat{\tau}]}{ } e^{-\beta \hat{T}(t)} f(\alpha) \hat{m}(d \alpha, d t) .
\end{aligned}
$$

Now suppose that $\hat{\tau}<\infty$. Let $\tau^{*}$ denote the first point of increase of $\hat{T}$ in $\left[\hat{\tau}, \hat{\tau}_{1}\right]$. More precisely, let $\tau^{*} \doteq \inf \{t \in[\hat{\tau}, \infty): \hat{T}(t+\delta)>\hat{T}(t)$ for all $\delta>0\} \wedge \hat{\tau}_{1}$. Note that

$$
\begin{aligned}
\mathbb{E} & 1_{\{\hat{\tau}<\infty\}} e_{\left(\hat{\tau}, \hat{\tau}_{1}\right]} e^{-\beta \hat{T}(t)} \underset{[0, p]}{f(\alpha) d \hat{m}_{t}(d \alpha) d \hat{T}(t)} \\
= & \mathbb{E} 1_{\{\hat{\tau}<\infty\}} e_{\left[\tau^{*}, \hat{\tau}_{1}\right]} e_{[0, p]}^{-\beta \hat{T}(t)} f(\alpha) d \hat{m}_{t}(d \alpha) d \hat{T}(t) .
\end{aligned}
$$

We now show that the above quantity is equal to 0 . Consider the evolution of the process $\hat{Z}$ over the interval $\left[\hat{\tau}, \tau^{*}\right]$. By definition, $\hat{Z}(\hat{\tau}) \notin \mathbb{S}^{o}$. Since $\hat{T}$ is constant over 
this time interval, we see from Theorem 5.4(2),(3) that $\hat{B}_{1}$ and $\hat{S}$ are both constant over this interval, and since neither $v_{1}$ nor $v_{2}$ can push the process into the interior of $\mathbb{S}$, we see that $\hat{Z}\left(\tau^{*}\right) \notin \mathbb{S}^{o}$. Define $s^{*} \doteq \hat{T}\left(\tau^{*}\right)$. Since $\tau^{*}$ is a point of increase of $\hat{T}$ we have $T\left(s^{*}\right)=T\left(\hat{T}\left(\tau^{*}\right)\right)=\tau^{*}$. Thus $Z\left(s^{*}\right)=\hat{Z}\left(\tau^{*}\right) \notin \mathbb{S}^{o}$, where $Z$ is defined by (47). Consider first the case $Z\left(s^{*}\right)=0$; then from (48), $\left\langle S_{2}\right\rangle$ is strictly increasing at $s^{*}$. From this it follows that for all $\delta>0$ there exists $s_{\delta} \in\left[s^{*}, s^{*}+\delta\right]$ such that $\operatorname{dist}\left(Z\left(s_{\delta}\right), \mathbb{S}\right)>0$, i.e., $\operatorname{dist}\left(\hat{Z}\left(T\left(s_{\delta}\right)\right), \mathbb{S}\right)>0$. Now since $\mathbb{S}^{h} \rightarrow \mathbb{S}$ and $\hat{Z}^{h} \rightarrow \hat{Z}$ as $h \rightarrow 0$ we have $\operatorname{dist}\left(\hat{Z}^{h}\left(T\left(s_{\delta}\right)\right), \mathbb{S}^{h}\right)>0$ for all $h$ small enough. Therefore, by definition of $\hat{\tau}_{1}^{h}$ we must have $\hat{\tau}_{1}^{h} \leq T\left(s_{\delta}\right)$ for all $h$ small enough. This implies $\hat{\tau}_{1} \leq T\left(s_{\delta}\right)$. Now, taking $\delta \rightarrow 0$ and using the right continuity of $T$ at $s^{*}$ it follows that $\hat{\tau}_{1} \leq T\left(s^{*}\right)=\tau^{*}$. Hence the quantity in (57) is equal to 0 .

Finally, consider the case when $Z\left(s^{*}\right)=0$ (and $\left.\hat{\tau}<\infty\right)$. Let $s^{* *} \doteq \inf \{s>$ $\left.s^{*} \mid Z\left(s^{*}\right)=0\right\}$. From the dynamics of $Z$ (see (47)) it follows that for every $\delta>$ 0 , there exists $s_{\delta} \in\left[s^{* *}, s^{* *}+\delta\right]$ such that $\operatorname{dist}\left(Z\left(s_{\delta}\right), \mathbb{S}\right)>0$. Arguing as before, we have $\hat{\tau}_{1} \leq T\left(s^{* *}\right)$. Define $m_{t}(d \alpha) \doteq \hat{m}_{T(t)}(d \alpha)$ for $t \geq 0$. Since $C(t)=0$ for $t \in\left[s^{*}, s^{* *}\right]$ we get that $m_{t}=\delta_{0}$ for $t$ in this interval. Thus since $f(0)=0$, we have $\int_{[0, p]} f(\alpha) d m_{t}(d \alpha)=0$ for all $t \in\left[s^{*}, s^{* *}\right]$. Combining this with the fact that $\left[\tau^{*}, \hat{\tau}_{1}\right] \subset\left[T\left(s^{*}\right), T\left(s^{* *}\right)\right]$ we now see that the expression in (57) is 0 . Thus

$$
\begin{aligned}
& \mathbb{E} 1_{\{\hat{\tau}<\infty\}} e_{[0, p] \times\left[0, \hat{\tau}_{1}\right]} e^{-\beta \hat{T}(t)} f(\alpha) \hat{m}(d \alpha, d t) \\
& =\mathbb{E} 1_{\{\hat{\tau}<\infty\}} e_{[0, p] \times[0, \hat{\tau}]} e^{-\beta \hat{T}(t)} f(\alpha) \hat{m}(d \alpha, d t) .
\end{aligned}
$$

Combining (55), (56), and (58) we now get

$$
\limsup _{h \rightarrow 0} J^{h}\left(z_{h}, U^{h}\right) \leq \mathbb{E} e_{[0, \hat{\tau}]} e^{-\beta \hat{T}(t)} \quad f(\alpha) d \hat{m}_{t}(d \alpha) \quad d \hat{T}(t) .
$$

We next consider the time inversion. Recall that $\tau \doteq \inf \left\{t: Z(t) \notin \mathbb{S}^{o}\right\}$. Note that $\tau \geq \hat{T}(\hat{\tau})$. Using this inequality and Lemma 4.3 we have

$$
\mathbb{E}_{[0, \hat{\tau}]} e^{-\beta \hat{T}(t)} \quad{ }_{[0, p]} f(\alpha) d \hat{m}_{t}(d \alpha) \quad d \hat{T}(t) \leq \mathbb{E} \mathbb{[ 0 , \tau ]}_{[0, p]} e^{-\beta t} \quad f(\alpha) d m_{t}(d \alpha) \quad d t .
$$

Inequality (53) now follows from the above inequality and Remark 5.7.

We now proceed to the proof of the reverse inequality

$$
\liminf _{h \rightarrow 0} V^{h}\left(z_{h}\right) \geq V_{\ell, p}(z)
$$

We begin with the following lemma which allows us to restrict our attention to controls that have several simplifying features. The proof is contained in the appendix. Recall the definition of an admissible control above Proposition 2.2 and the corresponding cost defined above (9).

Lemma 5.9. Let $\Phi$ be a probability system and $U \in \mathcal{A}_{p}(\Phi, z)$ be a control with corresponding cost function $J_{\ell}(z, U)$. Then for any $\delta>0$ there exists $U_{\delta} \in \mathcal{A}_{p}(\Phi, z)$ such that $\left|J_{\ell}(z, U)-J_{\ell}\left(z, U_{\delta}\right)\right|<\delta$ and $U_{\delta}$ satisfies the following:

1. There exists $T \in(0, \infty)$ such that $M_{\delta}(t)=M_{\delta}(T), N_{\delta}(t)=N_{\delta}(T)$, and $C_{\delta}(t)=0$ for all $t \geq T$. 
2. There exists $L \in(0, \infty)$ such that

$$
\sup _{0 \leq t<\infty} \sup _{\omega}\left(M_{\delta}(t \wedge \tau, \omega)+N_{\delta}(t \wedge \tau, \omega)\right) \leq L .
$$

3. There exist $\eta, \theta \in(0, \infty)$ and $K \in \mathbb{N}$ such that $C(t), M(t), N(t)$ take values in the finite set $\{k \eta: k=0,1,2, \ldots, K\}$. Furthermore, $C, M$, and $N$ are piecewise constant with possible time points of change being $\{0, \theta, 2 \theta, 3 \theta, \ldots\}$.

4. There exists a $\gamma \in(0, \infty)$ such that $\theta$ is an integer multiple of $\gamma$ and the chosen control $U=(C, M, N)$ satisfies the following equality for $m \geq 1$ :

$$
\begin{aligned}
& \mathbb{P}[(C(m \theta), \delta M(m \theta), \delta N(m \theta))=k \eta \mid U(s), s<m \theta ; W(s), s \leq m \theta] \\
& \quad=\mathbb{P}[(C(m \theta), \delta M(m \theta), \delta N(m \theta))=k \eta \mid U(n \theta), n<m ; W(l \gamma), l \gamma \leq m \theta],
\end{aligned}
$$

where $k=\left(k_{1}, k_{2}, k_{3}\right)$ and $k_{1}, k_{2}, k_{3}$ are integers, at most one of which is nonzero.

5. Denoting for $m \geq 1, \Psi(m) \doteq\{C(n \theta), \delta M(n \theta), \delta N(n \theta), n<m\}, \mathcal{W}(m) \doteq$ $\{W(l \gamma), l \gamma \leq m \theta\}$, and $k \doteq\left(k_{1}, k_{2}, k_{3}\right)$, rewrite the above probability as

$$
\begin{aligned}
& \mathbb{P}\left[C(m \theta)=k_{1} \eta, \delta M(m \theta)=k_{2} \eta, \delta N(m \theta)=k_{3} \eta \mid \Psi(m), \mathcal{W}(m)\right] \\
& \quad \doteq q_{m, k}(\Psi(m), z, \mathcal{W}(m)) .
\end{aligned}
$$

Denote $\mathbb{P}\left[U(0)=\left(k_{1} \eta, k_{2} \eta, k_{3} \eta\right)\right]$ by $q_{0, k}(z)$. For each $m \geq 0$, the function $q_{m, k}$ can be chosen so that the function $(z, w) \rightarrow q_{m, k}(\psi, z, w)$ is continuous for every $\psi$.

Construction of asymptotically near optimal admissible controls for the MDP. Fix a probability system $\Phi, z \in \mathbb{S}_{\ell}$, and a sequence $\left\{z_{h}\right\}$ such that $z_{h} \in \mathbb{S}^{h}$ and $z_{h} \rightarrow z$ as $h \rightarrow 0$. Let $\epsilon>0$ be arbitrary. Let $U \in \mathcal{A}_{p}(\Phi, z)$ be such that $U$ satisfies properties $1-5$ of Lemma 5.9 and $V_{\ell, p}(z)-\epsilon \leq J_{\ell}(z, U)$. For each $h>0$, we construct from $U$ an admissible control sequence $\left\{U_{n}^{h}, n \geq 0\right\}$ for the MDP in Definition 3.1 with initial condition $z_{h}$ such that the cost for $U^{h}$ asymptotically agrees with the cost of $U$. We outline below the main steps in the construction of such a control sequence. Let $\mathcal{K} \doteq\left\{\left(k_{1}, k_{2}, k_{3}\right): k_{i}=0,1, \ldots, K ; i=\right.$ $1,2,3$ such that at most one of $k_{1}, k_{2}, k_{3}$ is positive $\}$.

Step 1 . We begin by taking a random draw, denoted by $\kappa=\left(\kappa_{1}, \kappa_{2}, \kappa_{3}\right)$, from the probability distribution $\left\{q_{0, k}\left(z_{h}\right), k \in \mathcal{K}\right\}$. Set $\tilde{U}_{0}^{h}=\kappa \eta, Z_{0}^{h}=z_{h}$, and $\Psi^{h}(1) \doteq \tilde{U}_{0}^{h}$. Also set $\tilde{n}_{0}=0$. Note that at most one of $\kappa_{2}$ and $\kappa_{3}$ will be nonzero. If both $\kappa_{2}$ and $\kappa_{3}$ are 0 , set $n_{1}=0$, skip Step 2 below and go to Step 3. Otherwise proceed to Step 2 .

Step 2(A). Recall the kernel $p_{h}$ defined in (21). If $\kappa_{2}>0$, let $U_{0}^{h}=(1,0)$ and take a random draw, denoted by $Z_{1}^{h}$, from $p_{h}\left(Z_{0}^{h}, U_{0}^{h}, d \tilde{z}\right)$. We express this as "the chain takes a purchase control step." If $Z_{1}^{h} \in \partial_{\mathrm{R}}^{h}$, we set $U_{1}^{h}=(3,0)$ and draw $Z_{2}^{h}$ from $p_{h}\left(Z_{1}^{h}, U_{1}^{h}, d \tilde{z}\right)$; i.e., the chain takes a reflection step. Otherwise set $U_{1}^{h}=(1,0)$ and draw $Z_{2}^{h}$ from $p_{h}\left(Z_{1}^{h}, U_{1}^{h}, d \tilde{z}\right)$. Define $\left(U_{n}^{h}, Z_{n}^{h}\right), n=1,2, \ldots$, recursively by taking either a purchase control step or, if needed, a reflection step, until a total of $\left[\kappa_{2} \eta / h\right]$ purchase control steps have been taken. Denote by $n_{1}$ the index of the state after the last purchase control has been exercised.

Step 2(B). If $\kappa_{3}>0$, let $U_{0}^{h}=(2,0)$ (that is, the chain takes a sales control step) and proceed as in Step 2(A) above, alternating sales control steps and reflection 
steps (when needed) until $\left[\kappa_{3} \eta / h\right]$ sales control steps have been taken. Again, let $n_{1}$ denote the index of the state after the last sales control has been exercised.

Step 3. If $Z_{n_{1}}^{h} \in \partial_{\mathrm{R}}^{h}$, set $U_{n_{1}}^{h}=(3,0)$, and the chain takes a reflection step. Otherwise set $U_{n_{1}}^{h}=\left(0, \kappa_{1} \eta\right)$ and draw $Z_{n_{1}+1}^{h}$ from $p_{h}\left(Z_{n_{1}}^{h}, U_{n_{1}}^{h}, d \tilde{z}\right)$; i.e., the chain takes a diffusion step with $c=\kappa_{1} \eta$. Let $t_{n}^{h}$ be as defined below (23). Define $\left(U_{n}^{h}, Z_{n+1}^{h}, n=n_{1}, n_{1}+1, n_{1}+2, \ldots, \tilde{n}_{1}-1\right.$ recursively, where $\tilde{n}_{1} \doteq \inf \left\{n: t_{n}^{h} \geq \theta\right\}$, as follows. If $Z_{n}^{h} \in \partial_{\mathrm{R}}^{h}$, set $U_{n}^{h}=(3,0)$; otherwise set it to be $\left(0, \kappa_{1} \eta\right)$. Draw $Z_{n+1}^{h}$ from $p_{h}\left(Z_{n}^{h}, U_{n}^{h}, d \tilde{z}\right)$.

Step 4. Next we define the "pre-Wiener process" that is needed to obtain the control at the next step. Let $\left\{\nu_{n}, n \geq 1\right\}$ be an independent, identically distributed (i.i.d.) sequence of $N(0,1)$ random variables, independent of $\left(U_{n}^{h}, Z_{n+1}^{h}\right)_{n=0}^{\tilde{n}_{1}-1}$. Define $S_{n}^{h}$ for $n \leq \tilde{n}_{1}-1$ as in (26); here we consider only the second component $S_{n, 2}^{h}$. Set $\mathcal{S}_{0}^{h} \equiv 0$, and for $\tilde{n}_{0}<n \leq \tilde{n}_{1}-1$,

$$
\mathcal{S}_{n}^{h} \doteq \frac{S_{n+1,2}^{h}-S_{n, 2}^{h}}{\sigma Y_{n}^{h}} 1_{\left\{\left|Y_{n}^{h}\right| \neq 0\right\}}+\nu_{n} \sqrt{\Delta_{n}^{h}} 1_{\left\{\left|Y_{n}^{h}\right|=0\right\}} .
$$

Next define $W_{\tilde{n}_{0}}^{h}=0$ and $W_{n}^{h} \doteq W_{\tilde{n}_{0}}^{h}+{ }_{i=0}^{n-1} \mathcal{S}_{i}^{h}$. Now define for $0 \leq t \leq \theta$,

$$
W^{h}(t) \doteq W_{n^{h}(t)}^{h}-W_{\tilde{n}_{0}}^{h} .
$$

Finally, define $\mathcal{W}^{h}(1) \doteq\left\{W^{h}(l \gamma), l \in \mathbb{N}_{0}, l \gamma \leq \theta\right\}$.

Step 5. Suppose we have, for $j=1, \ldots, m$, defined $\tilde{n}_{j} \doteq \inf \left\{n: t_{n}^{h} \geq j \theta\right\}$; $\left(Z_{n+1}^{h}, U_{n}^{h}\right), n=0,1, \ldots, \tilde{n}_{j}-1 ; \Psi^{h}(j)$; and $\mathcal{W}^{h}(j)$. Consider now the case $j=m+1$. Take a random draw, denoted once more by $\kappa=\left(\kappa_{1}, \kappa_{2}, \kappa_{3}\right)$, from $\left\{q_{m, k}\left(\Psi^{h}(m), z_{h}\right.\right.$, $\left.\left.\mathcal{W}^{h}(m)\right), k \in \mathcal{K}\right\}$. Set $\tilde{U}_{m}^{h}=\kappa \eta$ and $\Psi^{h}(m+1)=\left(\tilde{U}_{0}^{h}, \ldots, \tilde{U}_{m}^{h}\right)$. Follow Step 2 with $\tilde{U}_{0}^{h}$ replaced by $\tilde{U}_{m}^{h}$ and the starting index of $U^{h}$ replaced with $\tilde{n}_{m}$. Denote by $n_{m+1}$ the index of the state obtained after the last singular control step in Step 2. Follow Step 3 with $n_{1}$ replaced by $n_{m+1}$. Let $\tilde{n}_{m+1}=\inf \left\{n: t_{n}^{h} \geq(m+1) \theta\right\}$. This defines $\left(Z_{n+1}^{h}, U_{n}^{h}\right), i=0,1, \ldots, \tilde{n}_{m+1}-1$. Define $W^{h}(t)-W^{h}(m \theta)$, for $t \in[m \theta,(m+1) \theta)$, by the right side of $(62)$ as in Step 4 with $\tilde{n}_{0}, \tilde{n}_{1}$ replaced by $\tilde{n}_{m}, \tilde{n}_{m+1}$, respectively. Now set $\mathcal{W}^{h}(m+1) \doteq\left\{W^{h}(l \gamma), l \in \mathbb{N}, l \gamma \leq(m+1) \theta\right\}$.

Noting that $\tilde{n}_{m}$ is strictly increasing in $m$, we obtain the controlled chain $\left\{\left(Z_{n}^{h}, U_{n}^{h}\right)\right.$, $n=0,1,2, \ldots\}$ via the recursion:

$\left(\left\{\left(Z_{n+1}^{h}, U_{n}^{h}\right)\right\}_{n=0}^{\tilde{n}_{m}-1}, \Psi^{h}(m), \mathcal{W}^{h}(m)\right) \rightarrow\left(\left\{\left(Z_{n+1}^{h}, U_{n}^{h}\right)\right\}_{n=0}^{\tilde{n}_{m+1}-1}, \Psi^{h}(m+1), \mathcal{W}^{h}(m+1)\right)$.

The main step in the proof of (59) is showing that if interpolated processes $\left(Z^{h}, U^{h}\right)$ using the above control sequence are defined as below (25) and $W^{h}$ is defined by (62), then as $h \rightarrow 0$,

$$
\left(Z^{h}, U^{h}, W^{h}\right) \text { converges weakly in } D\left([0, \infty): \mathbb{R}^{5}\right) \text { to }(Z, U, W),
$$

where $W$ is a standard Brownian motion and $Z$ is defined by (7) with the initial condition $Z(0-)=z$. This convergence is established by proving convergence over the period $[j \theta,(j+1) \theta)$ for each $j$ in a recursive manner. Note that given the initial condition $Z(j \theta-)=z$ and the control value $U(j \theta)=k \eta$, the dynamics of $Z$ for $t \in[j \theta,(j+1) \theta)$ are particularly simple and are given as

$$
\begin{aligned}
& X(t)=x+(1-\mu) \kappa_{3} \eta-(1+\lambda) \kappa_{2} \eta+{ }_{j \theta}^{t}\left(r X(s)-\kappa_{1} \eta\right) d s, \\
& Y(t)=y+\kappa_{2} \eta-\kappa_{3} \eta+{ }_{j \theta}^{t} b Y(s) d s+{ }_{j \theta}^{t} \sigma Y(s) d W(s) .
\end{aligned}
$$


The following lemma provides the convergence of $\left(Z^{h}, U^{h}, W^{h}\right)$ over one fixed period $[j \theta,(j+1) \theta)$ given the initial data at $j \theta$. The proof follows via straightforward weak convergence arguments and thus is omitted.

Lemma 5.10. Fix $z \in \mathbb{S}_{\ell}$ and let $k=\left(k_{1}, k_{2}, k_{3}\right) \in \mathcal{K}$. Let $(Z(t), W(t))$ given on some probability system $\Phi$ be defined for $t \in[0, \theta]$ by $(7)$ with $Z(0-)=z$ and $(C(t), M(t), N(t))=k \eta$ for $t \in[0, \theta]$. Consider a sequence $\left\{z_{h}\right\}$ such that $z_{h} \in \mathbb{S}^{h}$ and $z_{h} \rightarrow z$ as $h \rightarrow 0$. Define $\tilde{n}_{1}$ and the sequence $\left\{U_{n}^{h}, Z_{n}^{h}\right\}_{n=0}^{\tilde{n}_{1}}$ via Steps 2 and 3 and $\left\{S_{n}^{h}, W_{n}^{h}\right\}_{n=0}^{\tilde{n}_{1}}$ by Step 4 . Let $\left\{\delta_{h}\right\}$ be a sequence of nonnegative reals such that $\delta_{h} \rightarrow 0$ as $h \rightarrow 0$. Define, for $t \in[0, \theta]$, the interpolated process $\left(Z^{h}, W^{h}, U^{h}, E^{h}\right)$ as before (see (62) and below (26)) with the change that $\Delta^{h}\left(Z_{0}^{h}, U_{0}^{h}\right)$ is replaced with $\delta_{h}+\Delta^{h}\left(Z_{0}^{h}, U_{0}^{h}\right)$. Denote the laws of $\left(Z^{h}, W^{h}, U^{h}\right)$ and $(Z, W, U)$ on $D\left([0, \theta]: \mathbb{R}^{5}\right)$ by $\Pi_{h}^{k, \delta_{h}}$ and $\Pi^{k}$, respectively. Then $\Pi_{h}^{k, \delta_{h}} \rightarrow \Pi^{k}$ as $h \rightarrow 0$.

In the following proposition we show that the cost, $J^{h}\left(z_{h}, U^{h}\right)$, corresponding to the above constructed control sequence, converges to $J_{\ell}(z, U)$ as $h \rightarrow 0$. The desired inequality in (59) then follows since $V^{h}\left(z_{h}\right) \geq J^{h}\left(z_{h}, U^{h}\right), J_{\ell}(z, U) \geq V_{\ell, p}(z)-\epsilon$, and $\epsilon>0$ is arbitrary.

Proposition 5.11. Let $\epsilon>0$ be arbitrary and fix $z \in \mathbb{S}_{\ell}$. Let $\Phi$ be a probability system and $U \equiv U(\epsilon) \in \mathcal{A}_{p}(\Phi, z)$ be such that $U$ satisfies properties $1-5$ of Lemma 5.9 and $V_{\ell, p}(z)-\epsilon \leq J_{\ell}(z, U)$. Let, for each $h>0,\left\{U_{n}^{h}\right\}$ be an admissible control sequence as constructed via Steps $1-5$ above. Then $J^{h}\left(z_{h}, U^{h}\right) \rightarrow J_{\ell}(z, U)$ as $h \rightarrow 0$, and consequently (59) holds.

Proof. For $t \geq 0$, let $n^{h}(t), Z^{h}(t), M^{h}(t), N^{h}(t), C^{h}(t)$ be as defined below (25). Define $U^{h} \equiv\left(M^{h}, N^{h}, C^{h}\right)$ and let $W^{h}$ be as in (62). We begin by establishing (63). Define for $j \in \mathbb{N}_{0}$ and $t \in[0, \theta)$,

$$
U_{j}^{h}(t) \doteq U^{h}(t+j \theta), Z_{j}^{h}(t) \doteq Z^{h}(t+j \theta), W_{j}^{h}(t) \doteq W^{h}(t+j \theta)-W^{h}(j \theta),
$$

and set $\left(U_{j}^{h}(\theta), Z_{j}^{h}(\theta), W_{j}^{h}(\theta)\right)=\left(U_{j}^{h}(\theta-), Z_{j}^{h}(\theta-), W_{j}^{h}(\theta-)\right)$. Define processes $U_{j}$, $Z_{j}, W_{j}, j \in \mathbb{N}_{0}$, in a similar manner. Recall the sequence $\left\{U_{j}^{h}\right\}$ constructed above Lemma 5.10 and let $\zeta_{j}^{h} \doteq\left(\tilde{U}_{j}^{h}, U_{j}^{h}, W_{j}^{h}, Z_{j}^{h}\right), \zeta_{j} \doteq\left(\tilde{U}_{j}, U_{j}, W_{j}, Z_{j}\right)$, where $\tilde{U}_{j} \doteq$ $(C(j \theta), \delta M(j \theta), \delta N(j \theta)), j \in \mathbb{N}_{0}$. Due to the piecewise constant feature of the control $U$, in order to prove (63), it suffices to show that

$$
\text { for all } n \in \mathbb{N}_{0}, \Upsilon_{h}^{n} \doteq\left\{\zeta_{j}^{h}\right\}_{j=0}^{n} \text { converges weakly to } \Upsilon \doteq\left\{\zeta_{j}\right\}_{j=0}^{n} \text { as } h \rightarrow 0 \text {. }
$$

We will prove (65) via induction (on $n$ ). The case $n=0$ is immediate from Lemma 5.10 and continuity of the kernel $q_{0, k}$ on noting that for $k \in \mathcal{K}$ and $E \in \mathcal{B}\left(D\left([0, \theta], \mathbb{R}^{5}\right)\right.$,

$$
\begin{gathered}
\mathbb{P}\left(\tilde{U}_{0}^{h}=k \eta,\left(U_{0}^{h}, W_{0}^{h}, Z_{0}^{h}\right) \in E\right)=q_{0, k}\left(z_{h}\right) \Pi_{h}^{k, 0}(E), \\
\mathbb{P}\left(\tilde{U}_{0}=k \eta,\left(U_{0}, W_{0}, Z_{0}\right) \in E\right)=q_{0, k}(z) \Pi^{k}(E) .
\end{gathered}
$$

Suppose now that (65) holds for $n=0, \ldots, m$ and consider the case $n=m+1$. Denote the law of $\Upsilon_{n}^{h}, \Upsilon_{n}$ by $\varpi_{n}^{h}$ and $\varpi$, respectively. By the induction hypothesis, $\varpi_{m}^{h} \rightarrow \varpi_{m}$ as $h \rightarrow 0$. Furthermore, $\varpi_{m+1}^{h}$ can be expanded in terms of $\varpi_{m}^{h}$ as follows:

$$
d \varpi_{m+1}^{h}\left(v_{m+1}\right)={ }_{k \in \mathcal{K}} q_{m+1, k}\left(z_{h}, \tilde{u}^{*, m}, w^{*, m}\right) \Pi_{h}^{k, \delta_{h}\left(v_{m}\right)}\left(\varsigma_{m+1}\right) d \varpi_{m}^{h}\left(v_{m}\right),
$$

where $v_{m}=\left\{\varsigma_{j}\right\}_{j=0}^{m} ; \varsigma_{j}=\left(\tilde{u}_{j}, u_{j}, w_{j}, z_{j}\right) ; \tilde{u}_{j}=k \eta, k \in \mathcal{K} ;\left(u_{j}, w_{j}, z_{j}\right) \in D\left([0, \theta]: \mathbb{R}^{5}\right)$; $\tilde{u}^{*, m}=\left\{\tilde{u}_{j}\right\}_{j=0}^{m} ; w^{*, m}=\left\{w_{j}(l \gamma), l \in \mathbb{N}, l \gamma \leq \theta\right\}_{j=0}^{m}$; and $\delta_{h}$ is a measurable map from the state space of $\Upsilon_{m}$ to $[0,1]$ satisfying $0 \leq \delta_{h}\left(v_{m}\right) \leq \tilde{\Delta}_{*}^{h}$. 
From the continuity properties of the kernel $\left\{q_{m+1, k}\right\}$ and the weak convergence of $\varpi_{m}$ to $\varpi$, we have for all continuous and bounded functions $F_{1}, F_{2}$ defined on suitable spaces, as $h \rightarrow 0$,

$$
\begin{gathered}
F_{1}\left(v_{m}\right) \underset{k \in \mathcal{K}}{ } q_{m+1, k}\left(z, \tilde{u}^{*, m}, w^{*, m}\right)\left(F_{2}\left(\zeta_{m+1}\right) d \Pi^{k}\left(\zeta_{m+1}\right)\right) d \varpi_{m}^{h}\left(v_{m}\right) \\
(67) \rightarrow \quad F_{1}\left(v_{m}\right) \underset{k \in \mathcal{K}}{\rightarrow} q_{m+1, k}\left(z, \tilde{u}^{*, m}, w^{*, m}\right)\left(F_{2}\left(\zeta_{m+1}\right) d \Pi^{k}\left(\zeta_{m+1}\right)\right) d \varpi_{m}\left(v_{m}\right) .
\end{gathered}
$$

Next, from Lemma 5.10, for all sequences $\left\{\delta_{h}\right\}$ converging to 0 and compact sets $E$ (of Euclidean space of appropriate dimension), as $h \rightarrow 0$,

$$
\begin{aligned}
& \sup _{k \in \mathcal{K}, \tilde{u}^{*, m} \in \eta \mathcal{K}^{m+1}, w^{*, m} \in E} \mid \quad F_{2}\left(\zeta_{m+1}\right) q_{m+1, k}\left(z_{h}, \tilde{u}^{*, m}, w^{*, m}\right) d \Pi_{h}^{k, \delta_{h}}\left(\zeta_{m+1}\right) \\
& -\quad F_{2}\left(\zeta_{m+1}\right) q_{m+1, k}\left(z_{h}, \tilde{u}^{*, m}, w^{*, m}\right) d \Pi^{k}\left(\zeta_{m+1}\right) \mid \rightarrow 0 .
\end{aligned}
$$

The weak convergence of $\varpi_{m+1}^{h}$ to $\varpi_{m+1}$ now follows on combining (66), (67), and (68). This proves (63).

We now address convergence of the cost functions. First, let $T$ be as in Lemma 5.9. Recall $\eta^{h}=\inf \left\{n \geq 0: Z_{n}^{h} \in \partial^{h}\right\}$ and $\tau^{h}=t_{\eta^{h}}^{h}$. Note that $\tau^{h}=\inf \{t \geq 0$ : $\left.Z^{h}(t) \in \partial^{h}\right\}$ due to the piecewise constant nature of $Z^{h}(t)$.

Let $\tau=\inf \left\{t \geq 0: Z(t) \notin \mathbb{S}^{o}\right\}$. It can be shown in a manner similar to that used in the proof of Lemma 5.1 that $\tau^{h} \rightarrow \tau$ as $h \rightarrow 0$ on the set $\{\tau=\infty\}$. Also, on the set $\{\tau<\infty\}$ for every $\delta>0$ there exists $t \in[\tau, \tau+\delta)$ and $\epsilon>0$ such that $\operatorname{dist}(Z(t), \mathbb{S})>\epsilon$. Furthermore, $\left|Z^{h}(t)-Z(t)\right|$ uniformly on $[0, T]$ and $\partial^{h} \rightarrow \partial \mathbb{S}$ as $h \rightarrow 0$. Together these three facts imply $\tau^{h} \wedge T \rightarrow \tau \wedge T$ as $h \rightarrow 0$. Therefore, since $\left(z_{h}, Z^{h}, U^{h}, W^{h}, \tau^{h}\right) \rightarrow(z, Z, U, W, \tau)$, by the dominated convergence theorem,

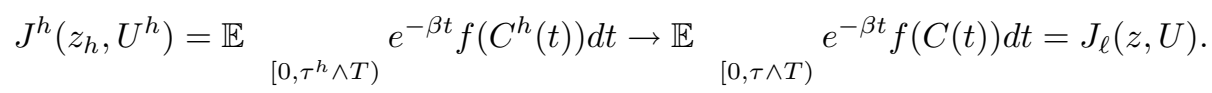

Combining Corollary 2.5 and Propositions 5.8 and 5.11 we have the following theorem.

THEOREM 5.12. Let $z \in \mathbb{S}$ and let $\left\{z_{h}, h>0\right\}$ be a sequence with $z_{h} \in \mathbb{S}_{\ell}^{h}$ such that $z_{h} \rightarrow z$ as $h \rightarrow 0$. Then $\lim _{p \rightarrow \infty} \lim _{\ell \rightarrow \infty} \lim _{h \rightarrow 0} V^{h}\left(z^{h}\right)=\lim _{p \rightarrow \infty} \lim _{\ell \rightarrow \infty} V_{\ell, p}(z)=$ $V(z)$.

6. Computational methods for the MDP. The convergence results in the previous section ensure that for small values of $h$, the MDP defined in section 3 provides a good approximation to the diffusion control problem defined in section 2 . In this section we outline the numerical methods for solving the MDP. Specifically, we provide the algorithm through which we compute the value function (25) and the associated optimal control for each initial state $z_{h} \in \mathbb{S}_{\ell}^{h}$. In practice, we fix a value of $h$ and use the associated MDP to provide approximations to the diffusion control problem. Thus, for the remainder of the section, we will take $h$ as a fixed value and suppress it in the notation.

Specifying the controlled Markov chain. In section 3, we specified a choice of transition probabilities and interpolation intervals which satisfy the local consistency criteria; see (15)-(17) and (20). Many variations of this choice are possible; when 
specifying the particular controlled Markov chain, consideration must be given to the numerical implementation. For example, note that neither the denominators of the probabilities nor the interpolation intervals in (15) depend on the value of $c$. This was accomplished by allowing the self-transition $(x, y)$ to $(x, y)$. Also, we have separated the pure diffusion effects from the effects of the consumption control. That is, as consumption always decreases wealth, we associate it with only the transition from $(x, y)$ to $(x-h, y)$. Recall that $\Delta(z, u)=0$ for all $z$ if $u=(3, c)$; that is, the interpolation interval is 0 if reflection occurs. Hence, using (24), a reflection step incurs no cost and thus $V(\ell+h, y)=V(\ell, y), V(x, \ell+h)=V(x, \ell)$. It is a consequence of Definition 3.1 that $\mathbb{P}\left[I_{n}=3 \mid Z_{n} \in \partial_{\mathrm{R}} \backslash \partial^{h}\right]=1$ for all $n$; that is, reflection is the only admissible action for states in the reflecting boundary. Therefore, by adjusting the transition probabilities associated with the diffusion and singular controls, it is possible to eliminate states in the reflecting boundary without affecting the cost function. This modification helps in speeding up the convergence of the numerical scheme.

In what follows, we will assume that the reflecting boundary states have been eliminated and the appropriate adjustments to the transition probabilities made. Thus, the state space of the controlled Markov chain used in the numerical schemes is given by $\mathbb{S}_{\mathrm{MDP}} \doteq \mathbb{S}_{\ell}^{h+} \backslash \partial_{\mathrm{R}}$, and the control space is $\mathcal{U}_{\mathrm{MDP}} \doteq\{0,1,2\} \times[0, p]$.

Dynamic programming equation. Let $U \equiv\left\{U_{n}, n=0,1,2, \ldots\right\}$ be an admissible control sequence (see Definition 3.1) for the MDP with state space $\mathbb{S}_{\mathrm{MDP}}$, control space $\mathcal{U}_{\mathrm{MDP}}$, and initial state $z$. For the numerical methods it is convenient to work with the cost function

$$
J(z, U)=\mathbb{E}{ }_{n=0}^{\eta-1} e^{-\beta t_{n}} f\left(C_{n}\right) \tilde{\Delta}\left(Z_{n}, C_{n}\right) 1_{\left\{I_{n}=0\right\}},
$$

which is asymptotically equivalent to (24). Recall that the value function is given as $V(z)=\sup _{U \in \mathcal{A}(z)} J(z, U)$.

We now present the dynamic programming equation that characterizes the value function. We begin by introducing the class of feedback controls. A feedback control is a measurable function $\mathbf{u}: \mathbb{S}_{\mathrm{MDP}} \rightarrow \mathcal{U}_{\mathrm{MDP}}$. We write $\mathbf{u}=(\mathbf{i}, \mathbf{c})$, where $\mathbf{i}$ and $\mathbf{c}$ are the two coordinates of the function $\mathbf{u}$. Using such a function one can construct an admissible control pair $\left(Z_{n}, U_{n}\right)$ recursively by setting $Z_{0}=z_{0}, U_{n}=\mathbf{u}\left(Z_{n}\right), n \geq 0$, and

$$
\mathbb{P}\left[Z_{n+1} \in \cdot \mid Z_{0}, \ldots, Z_{n}, U_{0}, \ldots, U_{n}\right]=p\left(Z_{n}, U_{n}, \cdot\right) .
$$

With an abuse of terminology we will refer to this sequence $\left\{U_{n}\right\}$ as a feedback control as well. Note that $U_{n} \equiv\left(I_{n}, C_{n}\right)=\left(\mathbf{i}\left(Z_{n}\right), \mathbf{c}\left(Z_{n}\right)\right)$.

If $U=\left\{U_{n}, n=0,1,2, \ldots\right\}$ is a feedback control, then one can easily check that the pair $\left(Z_{n}, U_{n}\right)$ is a Markov chain, from which it follows that for all $z \in \mathbb{S}_{\mathrm{MDP}}$,

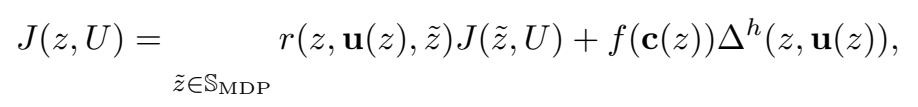

where $r(z, \mathbf{u}(z), \tilde{z})=e^{-\beta \Delta(z, \mathbf{u}(z))} p(z, \mathbf{u}(z), \tilde{z})$. Observing that $J(z, U)=0$ for all $z \in \partial^{h}$, the summation above can be taken over $\tilde{z} \in \mathbb{S}^{*} \doteq \mathbb{S}_{\mathrm{MDP}} \backslash \partial^{h}$.

We can write the above equality in matrix form as follows. Let $\left|\mathbb{S}^{*}\right|=s$ and fix an ordering of all the states in $\mathbb{S}^{*}$, i.e., $\mathbb{S}^{*}=\left\{z_{1}, \ldots, z_{s}\right\}$. Let $F(\mathbf{u})$ be an $s \times 1$ vector 
whose $i$ th entry is $f\left(\mathbf{c}\left(z_{i}\right)\right) \Delta\left(z_{i}, \mathbf{u}\left(z_{i}\right)\right), i=1, \ldots, s$. Let $R(\mathbf{u})$ be the $s \times s$ matrix with the $(i, j)$ th entry as $r\left(z_{i}, \mathbf{u}\left(z_{i}\right), z_{j}\right)$. Finally, let $J(\mathbf{u})$ be the $s \times 1$ vector with the $i$ th entry being $J\left(z_{i}, U\right)$. Then using these matrices, (70) can be written as

$$
J(\mathbf{u})=R(\mathbf{u}) J(\mathbf{u})+F(\mathbf{u}) .
$$

Next, for $u \in \mathcal{U}_{\mathrm{MDP}}$ let $R(u)$ be the $s \times s$ matrix with $(i, j)$ th entry $r\left(z_{i}, u, z_{j}\right)$. From standard arguments (cf. section 5.8 in [19]) it follows that the value function $V$ satisfies the following dynamic programming equation:

$$
V=\sup _{u \in \mathcal{U}_{\mathrm{MDP}}} R(u) V+F(u)
$$

where in the above equation $V$ is interpreted as an $s \times 1$ vector whose $i$ th entry is $V\left(z_{i}\right)$, and the supremum on the right-hand side above is taken row by row.

The following contraction property is central in the characterization of the value function via the dynamic programming equation in (72). The proof of the following lemma relies on the fact that the cost is of the discounted form with a strictly positive discount factor at all diffusion control steps, and although the discount is zero for singular control steps, such steps tend to push the process towards the boundary of the domain and thus cannot occur "too often."

Lemma 6.1. For all feedback controls $\mathbf{u}, R^{n}(\mathbf{u}) \rightarrow 0$ as $n \rightarrow \infty$.

Proof. Let $\mathbf{u}$ be a feedback control and denote $R^{n}(\mathbf{u})$ by simply $R^{n}$ with entries $r_{i j}^{n}, i, j=1, \ldots, s$. It suffices to show ${ }_{j=1}^{s} r_{i j}^{n} \rightarrow 0$ as $n \rightarrow \infty$, for each $i=1, \ldots, s$.

Let $\left(Z_{n}, U_{n}\right)$ be the controlled Markov chain associated with feedback control $\mathbf{u}$ and a transition kernel as defined in section 3 with the modifications discussed in this section, and let $\Delta_{k} \equiv \Delta\left(Z_{k}, U_{k}\right)$ be the associated interpolation intervals. Let $\eta \doteq \inf \left\{n: Z_{n} \in \partial^{h}\right\}$. A simple calculation yields for all $i=1, \ldots, s$ :

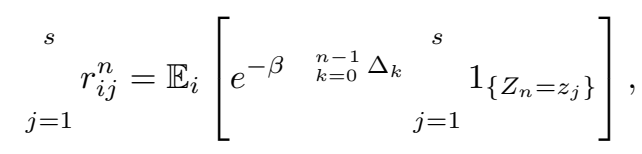

where $\mathbb{E}_{i}$ denotes the expectation given that $Z_{0}=z_{i}$.

Since the states in $\partial^{h}$ are not included in $\mathbb{S}^{*}$ and $p(z, \mathbf{u}(z), z)=1$ for $z \in \partial^{h}$, we have $1_{\left\{Z_{n}=z_{j}\right\}}=0$ for $j=1, \ldots, s$ when $n \geq \eta$. Thus we have

$$
{ }_{j=1}^{s} r_{i j}^{n}=\mathbb{E}_{i}\left[1_{\{n<\eta\}} e_{j=1}^{s} e^{-\beta}{ }_{k=0}^{n-1} \Delta_{k} 1_{\left\{Z_{n}=z_{j}\right\}}\right] .
$$

Fix $a \in \mathbb{Z}_{+}$; conditions on $a$ will be specified later. Define

$$
\tilde{d} \doteq \#\left\{\theta \in\{1,2, \ldots,[n / a]\}: U_{m}=(0, \cdot) \text { for some } m \in[(\theta-1) a, \theta a)\right\} .
$$

Set $\tilde{d}^{\prime}=[n / a]-\tilde{d}$. The integer $a$ is used to group the steps of the chain from 1 to $n$ together into intervals. The quantity $\tilde{d},\left(\tilde{d}^{\prime}\right)$ counts the number of such intervals with at least one diffusion step (respectively, no diffusion steps). By (14) there is a $\delta>0$ such that $\Delta_{n} \geq \delta$ for all diffusion steps (i.e., all $n$ such that $U_{n}=(0, \cdot)$ ). Also, recall that $\Delta_{n}=0$ if step $n$ is not a diffusion step. Combining these observations, we have

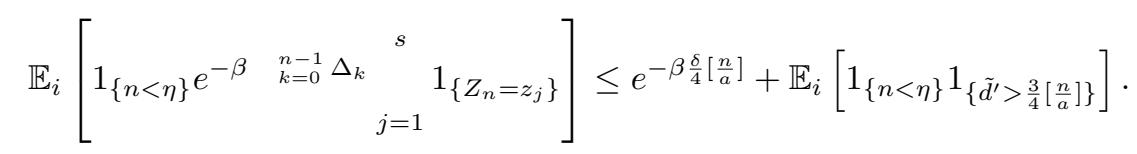


We will utilize the behavior of the singular controls to bound the second term on the right-hand side of the line above. Let $E_{1}, E_{2}, \ldots, E_{\tilde{d}^{\prime}}$ denote the intervals containing no diffusion steps, each of size $a$. Let $K_{d}$ denote the number of purchase control steps in $E_{d}$; then $a-K_{d}$ is the number of sales control steps in $E_{d}$. Due to the finiteness of the state space, the maximum number of successive transitions to the left is bounded; in particular, it is bounded by $B \doteq 2(\ell / h+1)$. Similarly, $B$ is a bound on the maximum number of downward transitions in a row. From (17) we see that each sales control always pushes the chain downward. Thus the application of too many sales controls in a row will cause the chain to hit the boundary. However, by (16) a purchase control potentially pushes the chain upward. Similarly, a purchase control always pushes the chain to the left, while a sales control has the potential to push the chain to the right. Thus in order to avoid hitting the boundary, the number of sales controls must be properly balanced by the number of purchase controls. More precisely, if $n<\eta$, we must have $\left|K_{d}-\left(a-K_{d}\right)\right|<B$; that is, $(a-B) / 2<K_{d}<(a+b) / 2, d=1, \ldots, \tilde{d}^{\prime}$. For $m \in E_{d}$ define $\tilde{L}_{m} \doteq 1_{\left\{Z_{m+1}-Z_{m}=(-h, 0)^{\prime}, U_{m}=(1,0)\right\}}$. The random variable $\tilde{L}_{m}$ indicates if the chain moves strictly to the left at step $m$ given that a purchase control is applied. Since on $E_{d}$ there are no diffusion steps and movement to the left is possible only at purchase control steps, the number of increments $\delta Z_{k}$ equal to $(-h, 0)^{\prime}$ on $E_{d}$ is given by $m \in E_{d} \tilde{L}_{m} \doteq L_{d}$.

Let $\epsilon$ be chosen to satisfy $0<\epsilon<p / 2$. Recall that at a purchase control step the chain moves to the left with probability $q \doteq \lambda /(1+\lambda)$. Thus by Cramer's theorem (see Theorem 2.1.24 in [8]) there exists a $\kappa \equiv \kappa(\epsilon)$ such that

$$
\mathbb{P}\left[L_{d}<K_{d}(q-\epsilon)\right] \leq \mathbb{P} \quad \frac{L_{d}}{K_{d}}-q>\epsilon \leq e^{-K_{d} \kappa} \leq e^{-\kappa(a-B) / 2},
$$

where the last inequality follows from the bound on $K_{d}$. We claim that for each $d=$ $1, \ldots, \tilde{d}^{\prime},\{\eta<n\} \cap\left\{L_{d}>q K_{d} / 2\right\}=\emptyset$. To see the claim suppose that $L_{d}>q K_{d} / 2$. Then the number of upward steps $\left(\delta Z_{k}=(\cdot, h)^{\prime}\right)$ in $E_{d}$, given by $K_{d}-L_{d}$, is at most $(1-q / 2) K_{d}$. The number of downward steps $\left(\delta Z_{k}=(\cdot,-h)^{\prime}\right)$ in $E_{d}$ equals the number of sales control steps, $a-K_{d}$. Thus using the bounds on $K_{d}$, we have

\#\{down steps in $\left.E_{d}\right\}-\#\left\{\right.$ up steps in $\left.E_{d}\right\} \geq a-K_{d}-(1-q / 2) K_{d} \geq a q / 4-(1+q / 4) B$,

which is greater than $B$ for $a>B(4+q) / q$. Henceforth fix such an $a$. On the other hand, on the set $\{\eta<n\}$ we must have $\mid \#\left\{\right.$ down steps in $\left.E_{d}\right\}-\#\left\{\right.$ up steps in $\left.E_{d}\right\} \mid<$ $B$; otherwise, the chain would hit the boundary. This leads to a contradiction and thus the claim holds. Combining this with (75) we have that

$$
\begin{aligned}
\mathbb{E}_{i}\left[1_{\{n<\eta\}} 1_{\left\{\tilde{d}^{\prime}>\frac{3}{4}\left[\frac{n}{a}\right]\right\}}\right] & =\mathbb{E}_{i}\left[1_{\left\{n<\eta, \tilde{d}^{\prime}>\frac{3}{4}\left[\frac{n}{a}\right]\right\}} \prod_{d=1}^{\tilde{d}^{\prime}} 1_{\left\{L_{d} \leq q K_{d} / 2\right\}}\right] \\
& \leq \mathbb{E}_{i}\left[\prod_{d=1}^{\frac{3}{4}\left[\frac{n}{a}\right]} 1_{\left\{L_{d} \leq q K_{d} / 2\right\}}\right] \\
& \leq e^{-\kappa \frac{a-B}{2} \frac{3}{4}\left[\frac{n}{a}\right]} .
\end{aligned}
$$

Finally, by (73), (74), and the above, we have ${ }_{j=1}^{s} r_{i j}^{n} \leq e^{-\beta \frac{\delta}{4}\left[\frac{n}{a}\right]}+e^{-\kappa \frac{a-B}{2} \frac{3}{4}\left[\frac{n}{a}\right]}$. The result now follows on noting that the term on the right approaches 0 as $n \rightarrow \infty$.

An immediate consequence of the lemma (cf. section 2.3 of [19]) is the following. 
TheOREM 6.2. For any feedback control $\mathbf{u}, J(\mathbf{u})$ is the unique solution to the equation $v=R(\mathbf{u}) v+F(\mathbf{u})$. Furthermore, the value function $\left\{V(z), z \in \mathbb{S}^{*}\right\}$ defined below (69) is the unique solution of (72). Denoting the arg max for the ith row maximization on the right side of (72) by $\mathbf{u}\left(z_{i}\right)$ and the control sequence corresponding to the feedback control $\mathbf{u}$ by $U=\left\{U_{n}, n=0,1,2, \ldots\right\}$, we have that $U$ is an optimal control, i.e., $J(z, U)=V(z)$ for all $z \in \mathbb{S}^{*}$.

From the above theorem it follows that in order to compute the value function and the optimal control it suffices to solve (72).

Numerical methods. We will use classical iterative methods to find the optimal control by solving the dynamic programming equation (72). A sketch of the algorithm is provided here. Details can be found in Chapter 6 of [19].

The following theorem provides the basis for the numerical approximation of the optimal control. We refer the reader to Theorem 6.2.1 in [19] for a proof.

TheOREm 6.3. Let $\mathbf{u}_{0}$ be a feedback control. Define a sequence of feedback controls $\left\{\mathbf{u}_{n}, n \geq 1\right\}$ and costs $\left\{J\left(\mathbf{u}_{n}\right), n \geq 1\right\}$ recursively as follows. Given $\mathbf{u}_{n}$, define

$$
\begin{aligned}
J\left(\mathbf{u}_{n}\right) & =R\left(\mathbf{u}_{n}\right) J\left(\mathbf{u}_{n}\right)+F\left(\mathbf{u}_{n}\right), \\
\mathbf{u}_{n+1} & \doteq \arg \max _{u \in \mathcal{U}_{\mathrm{MDP}}} R(u) J\left(\mathbf{u}_{n}\right)+F(u),
\end{aligned}
$$

where the arg max on the right-hand side is computed row by row. Then $J\left(\mathbf{u}_{n}\right) \rightarrow V$ as $n \rightarrow \infty$.

Given some control, (77) provides a way of "updating" the control in the search for the optimal control. However, this requires solving (76) to obtain the cost associated with the given control. Finding an exact solution to this equation can be numerically intensive since it involves the inversion of an $s \times s$ matrix. Thus we use instead an approximation to the cost function $J\left(\mathbf{u}_{n}\right)$ in (77). The following theorem provides a method for obtaining such an approximation. We refer the reader to Theorem 6.2.2 in [19] for the proof.

THEOREM 6.4. Let $\mathbf{u}$ be an admissible feedback control. Then for any initial $s \times 1$ vector $\tilde{J}_{0}$, the sequence defined recursively by

$$
\tilde{J}_{n+1}=R(\mathbf{u}) \tilde{J}_{n}+F(\mathbf{u})
$$

converges to $J(\mathbf{u})$.

The numerical method for finding the optimal control is obtained by combining Theorems 6.3 and 6.4 as follows.

Policy iteration: Having determined an approximation to $J\left(\mathbf{u}_{n}\right)$, denoted as $\tilde{J}\left(\mathbf{u}_{n}\right)$, one obtains $\mathbf{u}_{n+1}$ by solving the minimization problem in (77) by replacing $J\left(\mathbf{u}_{n}\right)$ there with $\tilde{J}\left(\mathbf{u}_{n}\right)$.

Value iteration: Given $\mathbf{u}_{n}$, iterate (78) a large number of times (say, $m$ ) with $R(\mathbf{u}$ ) there replaced by $R\left(\mathbf{u}_{n}\right)$ and initial value $\tilde{J}_{0}$ replaced by $\tilde{J}\left(\mathbf{u}_{n-1}\right)$. Set $\tilde{J}\left(\mathbf{u}_{n}\right) \doteq \tilde{J}_{m}$.

The numerical algorithm alternates between policy iterations and value iterations until some suitable stopping criterion is met. Several modifications of (78) are often used to improve numerical efficiency; see section 6.2.4 of [19] for details.

7. Numerical study. We now present the results of a small pilot study using the method described in section 6. We consider one of the examples in [31]. As in that reference, we set $r=0.07, b=0.12, \sigma=0.40$, and $\beta=0.10$. We consider the case $\lambda=\mu=0.01$ and the utility function $f(c)=2 \sqrt{c}$. We take $\ell=10$ as in [31] and $p=10$. The discretization parameter is taken to be $h=0.25$. (Note that [31] uses $h=0.025$.) 


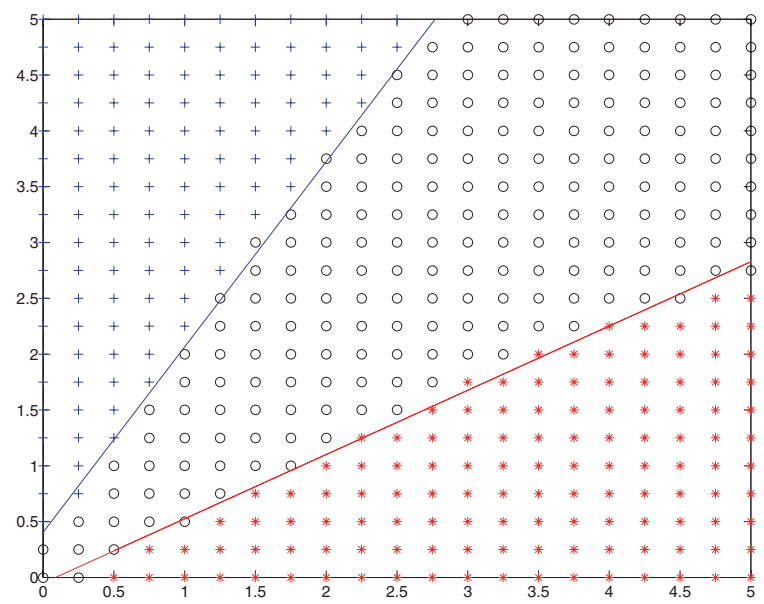

FIG. 2. Numerically computed optimal control.

To implement the numerical algorithm, we choose an initial feedback control matrix $\mathbf{u}_{0}$ given by, for $z \in \mathbb{S}^{*}$,

$$
\mathbf{u}_{0}(z)= \begin{cases}(0, p), & x \geq 0, y \geq 0 \\ (1,0), & x \geq 0, y<0 \\ (2,0), & \text { otherwise }\end{cases}
$$

Based on this control, the no-transaction region is the first quadrant of $\mathbb{R}^{2}$, and we always exercise the maximum amount of possible consumption. For $z \in \mathbb{S}^{*}$ we take $\tilde{J}_{0}(z)$ to be $75 \%$ of the value function computed in the absence of transaction costs; see equation (2.5) in [6].

We ran the algorithm described in the previous section. Figure 2 displays the first quadrant of the state space and illustrates the optimal control for this region. We see that the no-transaction region looks roughly like a cone. Consumption states are represented by the circles, purchase states by the plus signs, and sales states by the asterisks. The estimated boundaries of the no-transaction region (the solid lines in the figure) are given by the lines $y=0.575 x-0.050$ (the boundary of the "buy" region) and $y=1.659 x+0.405$ (the boundary of the "sell" region). The estimated sell boundary of the no-transaction region is similar to that obtained by Tourin and Zariphopoulou (see Figure 1 in [31]). However, the slope of our buy boundary appears to be lower than the slope illustrated in Figure 1 of [31]. A possible reason for this could be the difference in the discretization parameter. We used $h=0.25$ to produce the test results provided here. Within the no-transaction region, consumption remains at a fairly constant percentage of wealth, $11.5 \%$, which is very close to the constant percentage of consumption in the case of no-transaction costs (see Theorem 2.1 in [6]). We also compare the value function computed by the algorithm versus the value function in the case of no-transaction costs (again, see Theorem 2.1 in [6]). In general, the optimal value for an initial state computed in the presence of transaction costs is roughly $97 \%$ of the optimal value for the same state in the absence of transaction costs. However, when the initial wealth is small, this percentage tends to be lower (roughly $80 \%-90 \%$ ). 
8. Appendix. This section contains the proofs of Lemmas 4.4, 5.1, and 5.9. We refer the reader to the corresponding sections for the relevant notation and definitions.

Proof of Lemma 4.4. Without loss of generality, assume $h \in(0,1)$. Define

$$
Y_{i}^{h}(t) \doteq{ }_{k=0}^{n^{h}(t) \wedge \eta_{h}-1} \delta Z_{k}^{h} 1_{\left\{I_{k}^{h}=i\right\}}, \quad n_{i}^{h}(t) \doteq{ }_{k=0}^{n^{h}(t) \wedge \eta_{h}-1} 1_{\left\{I_{k}^{h}=i\right\}}, i=1,2 .
$$

Writing $Y_{i}^{h} \equiv\left(Y_{i, 1}^{h}, Y_{i, 2}^{h}\right)^{\prime}$, it follows from (16) and (17) that

$$
\mathbb{E} Y_{1,2}^{h}(t)=h \frac{1}{1+\lambda} \mathbb{E}\left[n_{1}^{h}(t)\right], \quad \mathbb{E} Y_{2,1}^{h}(t)=h(1-\mu) \mathbb{E}\left[n_{2}^{h}(t)\right] .
$$

A straightforward calculation shows $\left|B^{h}(t)\right| \leq c_{1}(1+t)$ and $\mathbb{E}\left|S^{h}(t)\right| \leq c_{2}(1+t)$, where the constants $c_{1}, c_{2}$ are independent of $h$ and $t$. From (16), (17) we see that $h n_{1}^{h}(t)=M^{h}(t)=Y_{1,1}^{h}(t)$ and $h n_{2}^{h}(t)=N^{h}(t)=Y_{2,2}^{h}(t)$. Thus from (28) there is $\tilde{c}_{1} \in(0, \infty)$ such that

$$
h n_{1}^{h}(t) \leq \tilde{c}_{1}(1+t)+\left|S_{1}^{h}(t)\right|+Y_{2,1}^{h}(t), h n_{2}^{h}(t) \leq \tilde{c}_{1}(1+t)+\left|S_{2}^{h}(t)\right|+Y_{1,2}^{h}(t) .
$$

Combining the above inequalities we have, for some $c_{3} \in(0, \infty), h \mathbb{E}\left[n_{1}^{h}(t)\right] \leq c_{3}(1+$ $t)+h(1-\mu) \mathbb{E}\left[n_{2}^{h}(t)\right]$ and $h \mathbb{E}\left[n_{2}^{h}(t)\right] \leq c_{3}(1+t)+h \mathbb{E}\left[n_{1}^{h}(t)\right] /(1+\lambda)$. It follows that $h \mathbb{E}\left[n_{1}^{h}(t)\right]$ and $h \mathbb{E}\left[n_{2}^{h}(t)\right]$ are "close" to each other. More precisely, there exist constants $\alpha \geq 1, c_{4}>0, L_{0}>0$ such that for $L \geq L_{0}$,

$$
h\left(\mathbb{E}\left[n_{1}^{h}(t)\right] \vee \mathbb{E}\left[n_{2}^{h}(t)\right]\right)>L \Rightarrow h\left(\mathbb{E}\left[n_{1}^{h}(t)\right] \wedge \mathbb{E}\left[n_{2}^{h}(t)\right]\right)>\alpha L-c_{4} .
$$

In particular, we have $\sup _{h} h \mathbb{E}\left[n_{1}^{h}(t)\right]=\infty$ if and only if $\sup _{h} h \mathbb{E}\left[n_{2}^{h}(t)\right]=\infty$. Now

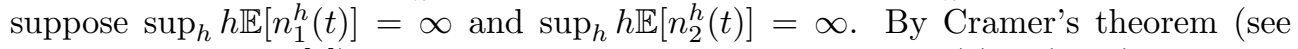
Theorem 2.1.24 in [8]), for all $\delta>0$ there exists a constant $c(\delta) \in(0, \infty)$ such that for all $k_{0} \in \mathbb{N}_{0}$ and $h>0$,

$$
\begin{aligned}
\max \{ & \mathbb{P}\left[\left|Y_{2,1}^{h}-h(1-\mu) n_{2}^{h}(t)\right|>\delta h n_{2}^{h}(t), n_{2}^{h}(t)=k_{0}\right], \\
& \left.\mathbb{P}\left[\left|Y_{1,2}^{h}-h(1 /(1+\lambda)) n_{1}^{h}(t)\right|>\delta h n_{1}^{h}(t), n_{1}^{h}(t)=k_{0}\right]\right\} \leq c(\delta) e^{-k_{0} c(\delta)} .
\end{aligned}
$$

Choose $\delta$ such that $\mu+\delta<1$ and $1 /(1+\lambda)-\delta>0$ (which is possible since $\mu \in(0,1)$ and $\lambda \in(0, \infty))$. Define $\alpha_{1}=1-(1-\mu-\delta)(1 /(1+\lambda)-\delta)<1$ and $\theta=\alpha_{1} / 4$. Fix $\epsilon \in(0,1)$ and choose $K$ large enough so that

$$
\frac{c(\delta)}{1-e^{-c(\delta)}} e^{-c(\delta)(K+1)}<\frac{\epsilon}{8} \text { and } \frac{c_{2}(1+t)}{\theta K-\tilde{c}_{1}(1+t)}<\frac{\epsilon}{8}
$$

Since by assumption, $\sup _{h} h \mathbb{E}\left[n_{1}^{h}(t)\right]=\sup _{h} h \mathbb{E}\left[n_{2}^{h}(t)\right]=\infty$, there exists $h^{\prime} \leq 1$ such that

$$
\mathbb{P} n_{1}^{h^{\prime}}(t)>\frac{K}{h^{\prime}}, n_{2}^{h^{\prime}}(t)>\frac{K}{h^{\prime}}>\epsilon
$$


Then for all $t \geq 0$,

$$
\begin{aligned}
& \mathbb{P}\left|Y_{2,1}^{h^{\prime}}(t)-h^{\prime}(1-\mu) n_{2}^{h^{\prime}}(t)\right|>\delta h^{\prime} n_{2}^{h^{\prime}}(t), n_{2}^{h^{\prime}}(t)>\frac{K}{h^{\prime}} \\
& =\sum_{j=\left[K / h^{\prime}\right]+1}^{\infty} \mathbb{P}\left[\left|Y_{2,1}^{h^{\prime}}(t)-h^{\prime}(1-\mu) n_{2}^{h^{\prime}}(t)\right|>\delta h^{\prime} n_{2}^{h^{\prime}}(t), n_{2}^{h^{\prime}}(t)=j\right] \\
& \leq \underbrace{\infty}_{j=\left[K / h^{\prime}\right]+1} c(\delta) e^{-c(\delta) j}=\frac{c(\delta)}{1-e^{-c(\delta)}} e^{-c(\delta)\left(\left[\frac{K}{h^{\prime}}\right]+1\right)}<\frac{\epsilon}{8},
\end{aligned}
$$

where the last inequality follows from the choice of $K$ in (80). Similarly, $\mathbb{P}\left[\mid Y_{1,2}^{h^{\prime}}(t)-\right.$ $\left.\frac{h^{\prime}}{1+\lambda} n_{1}^{h^{\prime}}(t) \mid>\delta h^{\prime} n_{1}^{h^{\prime}}(t), n_{1}^{h^{\prime}}(t)>\frac{K}{h^{\prime}}\right]<\frac{\epsilon}{8}$. Hence, in view of (81) we have

$$
\begin{gathered}
\mathbb{P} \min \left\{n_{1}^{h^{\prime}}(t), n_{2}^{h^{\prime}}(t)\right\}>\frac{K}{h^{\prime}},\left|Y_{2,1}^{h^{\prime}}(t)-h^{\prime}(1-\mu) n_{2}^{h^{\prime}}(t)\right| \leq \delta h^{\prime} n_{2}^{h^{\prime}}(t), \\
Y_{1,2}^{h^{\prime}}(t)-\frac{h^{\prime}}{1+\lambda} n_{1}^{h^{\prime}}(t) \leq \delta h^{\prime} n_{1}^{h^{\prime}}(t)>\frac{\epsilon}{2} .
\end{gathered}
$$

Let $E$ denote the event in the equation above. From (79) and (80),

$$
\begin{aligned}
\mathbb{P}\left[h^{\prime} n_{1}^{h^{\prime}}(t)-Y_{2,1}^{h^{\prime}}(t) \geq \theta K\right] & \leq \mathbb{P}\left[\left|S_{1}^{h^{\prime}}(t)\right| \geq \theta K-\tilde{c}_{1}(1+t)\right] \\
& \leq \frac{\mathbb{E}\left|S_{1}^{h^{\prime}}(t)\right|}{\theta K-\tilde{c}_{1}(1+t)} \\
& \leq \frac{c_{2}(1+t)}{\theta K-\tilde{c}_{1}(1+t)}<\frac{\epsilon}{8}
\end{aligned}
$$

Similarly, $\mathbb{P}\left[h^{\prime} n_{2}^{h^{\prime}}(t)-Y_{1,2}^{h^{\prime}}(t) \geq \theta K\right]<\frac{\epsilon}{8}$. Thus

$$
\begin{aligned}
\frac{\epsilon}{2}<\mathbb{P}[E] & \leq \mathbb{P}\left[E, h^{\prime} n_{1}^{h^{\prime}}(t)-Y_{2,1}^{h^{\prime}}(t)<\theta K, h^{\prime} n_{2}^{h^{\prime}}(t)-Y_{1,2}^{h^{\prime}}(t)<\theta K\right] \\
& +\mathbb{P}\left[h^{\prime} n_{1}^{h^{\prime}}(t)-Y_{2,1}^{h^{\prime}}(t) \geq \theta K\right]+\mathbb{P}\left[h^{\prime} n_{2}^{h^{\prime}}(t)-Y_{1,2}^{h^{\prime}}(t) \geq \theta K\right] \\
& \leq \mathbb{P}[\tilde{E}]+\frac{\epsilon}{8}+\frac{\epsilon}{8}
\end{aligned}
$$

where $\tilde{E}$ is the event in the first term on the right side above. It follows that $\mathbb{P}[\tilde{E}]>\epsilon / 4$ and thus $\tilde{E}$ is nonempty. Now for any $\omega \in \tilde{E}$, we have from the definition of $\tilde{E}$ that

$$
h^{\prime} n_{1}^{h^{\prime}}(t)-h^{\prime}(1-\mu-\delta) n_{2}^{h^{\prime}}(t)<\theta K, \quad h^{\prime} n_{2}^{h^{\prime}}(t)-h^{\prime}(1 /(1+\lambda)-\delta) n_{1}^{h^{\prime}}(t)<\theta K .
$$

A straightforward calculation using these inequalities shows that for such $\omega$,

$$
h^{\prime} n_{1}^{h^{\prime}}(t) \leq \frac{2 \theta}{1-(1-\mu-\delta)(1 /(1+\lambda)-\delta)} K=\frac{K}{2} .
$$

However, this contradicts the fact that $h^{\prime} n_{1}^{h^{\prime}}(t)>K$ on $\tilde{E}$. Thus we must have $\sup _{h} h \mathbb{E}\left[n_{1}^{h}(t)\right]<\infty, \sup _{h} h \mathbb{E}\left[n_{2}^{h}(t)\right]<\infty$. The result now follows on recalling that $M^{h}(t)=h n_{1}^{h}(t)$ and $N^{h}(t)=h n_{2}^{h}(t)$.

Proof of Lemma 5.1. The inequality $V(z) \leq \bar{V}(z)$ is immediate since every exact control can be expressed as a relaxed control. Consider now the reverse inequality. 
Let $\Phi$ be a probability system and $\bar{U}=(\bar{m}, M, N) \in \overline{\mathcal{A}}(\Phi, z)$ be such that $\bar{V}(z) \leq$ $\bar{J}(z, \bar{U})+\epsilon$. From the boundedness of the cost function it follows that, without loss of generality, we can assume that there is a $T \in(0, \infty)$ such that $M(t)=M(t \wedge T)$ and $N(t)=N(t \wedge T)$ for all $t \in(0, \infty)$, and $\bar{m}_{t}(d \alpha)=\delta_{p}$ for all $t \geq T$. Also, $T$ can be chosen large enough so that $f_{*}(p) e^{-\beta T} / \beta<\epsilon$.

Let $Z$ be defined via (7) with $C(t) \doteq \int_{[0, p]} \alpha \bar{m}_{t}(d \alpha)$, and $\tau$ as before. Then

$$
\bar{J}(z, \bar{U}) \leq \mathbb{E} \underset{[0, p] \times[0, T \wedge \tau)}{ } e^{-\beta t} f(\alpha) m(d \alpha, d t)+\epsilon .
$$

Also, by modifying $m, M$, and $N$ if needed, we can assume that

$$
M(t)=M(T \wedge \tau), \quad N(t)=N(T \wedge \tau), \text { and } \bar{m}_{t}(d \alpha)=\delta_{p} \text { for all } t \geq T \wedge \tau .
$$

Following the proof of Theorem 1.2.1 in [2] one can show that there exists a sequence of exact controls $C_{n} \in \mathcal{A}_{p}(\Phi, z)$ which satisfy

$$
\sup _{0 \leq t \leq T_{1}} \quad{ }_{[0, p] \times[0, t]} e^{-\beta t} f(\alpha) m(d \alpha, d t)-{ }_{[0, t]} e^{-\beta t} f\left(C_{n}(t)\right) d t \rightarrow 0 \text { a.s., and }
$$

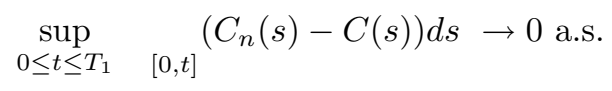

as $n \rightarrow \infty$ for all $T_{1} \in(0, \infty)$. In fact the cited theorem shows that, for each $n$, $C_{n}$ can be chosen such that it takes values in a finite set and there is a sequence $0<t_{1}^{n}<t_{2}^{n} \cdots$ such that $C_{n}$ is constant over $\left[t_{k}^{n}, t_{k+1}^{n}\right)$ for all $k \in \mathbb{N}_{0}$.

Let $Z_{n}$ be defined via (7) with $C$ replaced by $C_{n}$ and with $M$ and $N$ as introduced above. A straightforward application of Gronwall's inequality and (85) shows that for each $T_{1} \in(0, \infty)$, there is a $c \equiv c\left(T_{1}\right) \in(0, \infty)$ such that

$$
\sup _{0 \leq t \leq T_{1}} \mathbb{E}\left|Z_{n}(t)-Z(t)\right| \leq c \sup _{0 \leq t \leq T_{1}} \mathbb{E} \quad\left(C_{n}(s)-C(s)\right) d s
$$

Hence $Z_{n} \rightarrow Z$, in probability, uniformly on $[0, T]$.

If $\tau=\infty, Z(t) \in \mathbb{S}^{o}$ for all $t \geq 0$. Thus, (86) implies that there exists $N_{0}$ such that if $n>N_{0}$, then $Z_{n}(t) \in \mathbb{S}^{o}$ for all $t \geq 0$, and therefore $\tau_{n}=\infty$ for all $n>N_{0}$. Then clearly $\tau_{n} \rightarrow \tau$ a.s. as $n \rightarrow \infty$ on the set $\{\tau=\infty\}$. Next note that, a.s. on the set $\{\tau<\infty\}$ and for every $\delta>0$, there exist $t \in[\tau, \tau+\delta)$ and $\epsilon>0$ such that $\operatorname{dist}(Z(t), \mathbb{S})>\epsilon$. This is because, in view of $(83), Z(\tau+t), t \geq 0$, is described via (7) with initial condition $Z(\tau)$ and $M \equiv N \equiv 0$. If $Z(\tau)=0$, the property is satisfied trivially since $C(t)=p$ for all $t \geq \tau$. Otherwise, the property follows from a standard argument based on the law of the iterated logarithm for Brownian motion (cf. pages 260-261 in [19]). This, along with the convergence of $Z_{n}$ to $Z$, shows that $\tau_{n} \doteq \inf \left\{t: Z_{n}(t) \notin \mathbb{S}^{o}\right\}$ converges to $\tau$ a.s. as $n \rightarrow \infty$. In proving this statement we also use the observation that if $Z(t-) \notin \mathbb{S}$, then $Z(t) \notin \mathbb{S}$. Thus $\tau_{n} \wedge T \rightarrow \tau \wedge T$. Combining these observations with (84), we obtain

$$
\mathbb{E} \underset{\left[0, \tau_{n} \wedge T\right]}{ } e^{-\beta t} f\left(C_{n}(t)\right) d t \rightarrow \mathbb{E} \underset{[0, p] \times[0, \tau \wedge T]}{ } e^{-\beta t} f(\alpha) m(d \alpha, d t) .
$$


The result now follows on using this observation in (82).

Proof of Lemma 5.9. Let $\epsilon>0$ be arbitrary and let $T \in(0, \infty)$ be such that $f_{*}(p) e^{-\beta T} / \beta<\epsilon$. Consider $\tilde{U}=(\tilde{C}, \tilde{M}, \tilde{N})$ given by $\tilde{C}(t)=C(t) 1_{t<T}, \tilde{M}(t)=$ $M(t \wedge T)$, and $\tilde{N}(t)=N(t \wedge T), t \geq 0$. Clearly $\tilde{U} \in \mathcal{A}_{p}(z)$, and it is easy to check that $\left|J_{\ell}(z, U)-J_{\ell}(z, \tilde{U})\right|<\epsilon$. This proves 1 . Henceforth we will assume, without loss of generality, that 1 holds for $U$ in the statement of the lemma. Using (7) and the bounds on the state and control space, it is easy to $\operatorname{show}_{\sup _{0 \leq t \leq T}}[M(t \wedge \tau, \omega)+N(t \wedge \tau, \omega)] \leq$ $c_{1}+c_{2} \sup _{0 \leq t \leq T}|W(t)|$, where $c_{1}$ and $c_{2}$ are nonnegative constants that may depend on $T$. Let $L \in(0, \infty)$ be large enough so that $c_{2} \mathbb{E} \sup _{0 \leq t \leq T}|W(t)| /\left(L-c_{1}\right)<\epsilon$. Define $\tilde{U}$ by $\tilde{C} \equiv C, \tilde{M}(t) \doteq M(t) \wedge L, \tilde{N}(t) \doteq N(t) \wedge L$. Let $\tilde{Z}$ be the corresponding controlled process and $\tilde{\tau}$ the corresponding hitting time. Let $T$ be as in part 1, and define $A \doteq\left\{\sup _{0 \leq t \leq T}[M(t \wedge \tau)+N(t \wedge \tau)]<L\right\}$. Then

$$
J_{\ell}(z, \tilde{U})=\mathbb{E} \quad 1_{A} e_{[0, \tilde{\tau}]} e^{-\beta t} f(\tilde{C}(t)) d t+\mathbb{E} 1_{A^{c}} e_{[0, \tilde{\tau}]}^{-\beta t} f(\tilde{C}(t)) d t
$$

Using the bound on $f$, the choice of $L$, and Markov's inequality, the second term on the right side of the above inequality is bounded by $\epsilon$. Also, since on the set $A$, $M(t)<L$ and $N(t)<L$ for all $t \leq \tau \wedge T$, we have that the evolution of $\tilde{Z}$ is the same as that of $Z$. Therefore $\tilde{\tau}$ in the first expression on the right side of (88) can be replaced with $\tau$. This shows that $\left|J_{\ell}(z, \tilde{U})-J_{\ell}(z, U)\right| \leq 2 \epsilon$ and hence 2 follows.

We now consider 3 . Let $U \equiv(C, M, N)$ be an admissible control satisfying properties 1 and 2 and let $Z$ be the solution to (7) under $(C, M, N)$ defined on some probability system. Following Theorem 1.2.1 of [2] (see comments below (85)) we can assume without loss of generality that $C$ takes values in a finite set, is RCLL, and piecewise constant with finitely many points of change over $[0, T]$. We also assume without loss of generality (by modifying controls if needed) that $M(t)=M(t \wedge \tau)$, $N(t)=N(t \wedge \tau)$, and $C(t)=C(t) 1_{t<\tau}+p 1_{t \geq \tau}$. Fix $\eta, \theta \in(0, \infty)$ and define the piecewise constant processes $C_{\eta, \theta}, M_{\eta, \theta}$, and $N_{\eta, \theta}$ as follows. For $m=0$, define $M_{\eta, \theta}(m \theta)=M_{\eta, \theta}(0)=k \eta$ if $M(0) \in[k \eta, k \eta+\eta)$. For $m \geq 1$, set $\delta M_{\eta, \theta}(m \theta)=k \eta$ if $M(m \theta)-M(m \theta-\theta) \in[k \eta, k \eta+\eta)$. By property 2, we need only consider the finite set $\{k \eta: k=0,1,2, \ldots, K\}$, where $K$ is some positive integer. Then let $M_{\eta, \theta}(t)=M_{\eta, \theta}(m \theta)$ for $t \in[m \theta, m \theta+\theta)$. Define $N_{\eta, \theta}$ analogously based on $N$. Define $C_{\eta, \theta}(m \theta)=k \eta$ if $C(m \theta) \in[k \eta, k \eta+\eta)$ and $C_{\eta, \theta}(t)=C_{\eta, \theta}(m \theta)$ for $t \in[m \theta, m \theta+\theta)$. The constructed process $U_{\eta, \theta} \equiv\left(C_{\eta, \theta}, M_{\eta, \theta}, N_{\eta, \theta}\right)$ is an admissible control. Let $Z_{\eta, \theta}$ denote the solution to (7) under this control, defined on some probability system, and let $\tau_{\eta, \theta}$ denote the first time this process exits $\mathbb{S}_{\ell}^{o}$. Choose a sequence $\left(\eta_{k}, \theta_{k}\right)$ such that $\eta_{k}, \theta_{k} \rightarrow 0$ as $k \rightarrow \infty$. Denote $Z_{\eta_{k}, \theta_{k}}$ by $Z_{k}$. Similar abbreviations are used for $U_{\eta_{k}, \theta_{k}}, \tau_{\eta_{k}, \theta_{k}}$. One can easily check that $\left(Z_{k}, U_{k}\right) \rightarrow(Z, U)$ in $D\left([0, T], \mathbb{R}^{2} \times[0, p] \times[0, L] \times[0, L]\right)$ (in probability) as $k \rightarrow \infty$. If $\tau=\infty$, the uniform convergence $Z_{k} \rightarrow Z$ implies that there exists $K_{0}$ such that for all $k>K_{0}$ we have $Z_{k}(t) \in \mathbb{S}^{o}$ for all $t \geq 0$, and thus $\tau_{k}=\infty$ for all $k>K_{0}$. Therefore $\tau_{k} \rightarrow \tau$ a.s. as $k \rightarrow \infty$ on the set $\{\tau=\infty\}$. Next note that, a.s. on the set $\{\tau<\infty\}$, for every $\delta>0$ there exists $t \in[\tau, \tau+\delta)$ and $\epsilon>0$ such that $\operatorname{dist}(Z(t), \mathbb{S})>\epsilon$. This is because, on this set, by our choice of $U, Z(\tau+t), t \geq 0$, is described via (7) with $M \equiv N \equiv 0$ and initial condition $Z(\tau)$. In the case $Z(\tau)=0$ the property is satisfied trivially since $C(t)=p$ for all $t \geq \tau$. Otherwise, the property follows from an argument analogous to the proof of Theorem 9.4.3 of [19] (see pp. 260-261). Next, recalling that $Z_{k} \rightarrow Z, Z(t)$ is continuous for all $t \geq \tau$ and the observation that $Z(\tau-) \in\left(\mathbb{S}_{\ell}^{o}\right)^{c} \Rightarrow Z(\tau) \in\left(\mathbb{S}_{\ell}^{o}\right)^{c}$, 
we conclude $\tau_{k} \wedge T \rightarrow \tau \wedge T$ in probability. The convergence of $J\left(z, U_{k}\right)$ to $J(z, U)$ now follows. This proves 3 .

The proofs of 4 and 5 are quite standard and we provide only a sketch; the reader is referred to the proof of Theorem 10.3.1 of [19] (pages 285-287) for details. Assume that $U$ satisfies properties $1-3$, and let $\gamma>0$. Part 4 is essentially a consequence of the martingale convergence theorem on noting that the $\sigma$-fields $\mathcal{G}_{\gamma} \doteq \sigma\{U(n \theta), n<$ $m ; W(l \gamma), l \gamma \leq, \theta\}$ increase to the $\sigma$-field $\mathcal{G} \doteq \sigma\{U(n \theta), n<m ; W(s), s \leq, \theta\}$ as $\gamma \downarrow 0$. The main idea is to define controls $U_{\gamma}$ and controlled processes $Z_{\gamma}$ recursively over intervals $[m \theta,(m+1) \theta)$ by using the right side of $(60)$ in defining the law of $U_{\gamma}$ over $[m \theta,(m+1) \theta)$. Proving the weak convergence of $\left(Z_{\gamma}, C_{\gamma}\right)$ to $(Z, C)$ is straightforward. The convergence of hitting times is argued as in the proof of part 3. Finally, part 5 is proved by convolving $q_{m, k}$, defined in $(61)$, in the $(z, w)$ variables by a parametrized family of mollifiers and arguing weak convergence of the resulting controlled pair to $(Z, C)$ as the mollifying parameter approaches a suitable limit. Convergence of stopping times is argued, once more, as in 3.

Acknowledgments. The authors would like to thank the anonymous referees for their suggestions, which have greatly improved the presentation of the paper.

\section{REFERENCES}

[1] P. Billingsley, Convergence of Probability Measures, 2nd ed., John Wiley \& Sons, Inc., New York, 1999.

[2] V. S. Borkar, Control of Diffusion Processes, John Wiley \& Sons, Inc., New York, 1989.

[3] A. Budhiraja And A. GHosh, Diffusion approximations for controlled stochastic networks: An asymptotic bound for the value function, Ann. Appl. Probab., to appear.

[4] P.-L. Chow, J.-L. Menaldi, And M. Robin, Additive control of stochastic linear systems with finite horizon, SIAM J. Control Optim., 23 (1985), pp. 858-899.

[5] M. G. Crandall, H. Ishit, And P.-L. Lions, User's guide to viscosity solutions of second order partial differential equations, Bull. Amer. Math. Soc. (N. S.), 27 (1992), pp. 1-67.

[6] M. H. A. Davis And A. R. Norman, Portfolio selection with transaction costs, Math. Oper. Res., 15 (1990), pp. 676-713.

[7] P. Dupuis AND H. IsHiI, On Lipschitz continuity of the solution mapping to the Skorokhod problem, with applications, Stochastics/Stochastics Rep., 35 (1991), pp. 31-62.

[8] A. Dembo And O. Zeitouni, Large Deviations Techniques and Applications, Jones and Bartlett, Boston, 1993.

[9] S. N. Ethier And T. G. Kurtz, Markov Processes: Characterization and Convergence, John Wiley \& Sons, Inc., New York, 1986.

[10] B. G. Fitzpatrick And W. H. Fleming, Numerical methods for an optimal investmentconsumption model, Math. Oper. Res., 16 (1991), pp. 823-841.

[11] W. H. Fleming And H. M. Soner, Controlled Markov Processes and Viscosity Solutions, Springer-Verlag, New York, 1992.

[12] J. M. HARrison, Brownian models of queueing networks with heterogeneous customer population, in Stochastic Differential Systems, Stochastic Control Theory, and Their Applications, Springer, New York, 1988, pp. 147-186.

[13] J. M. Harrison and M. I. Reiman, Reflected Brownian motion on an orthant, Ann. Probab., 9 (1981), pp. 302-308.

[14] A. Hindy, C. HuAng, And S. Zhu, Numerical analysis of a free-boundary singular control problem in financial economics, J. Econom. Dynam. Control, 21 (1997), pp. 297-327.

[15] H. ISHI AND S. KoIKE, Boundary regularity and uniqueness for an elliptic equation with gradient constraint, Comm. Partial Differential Equations, 8 (1983), pp. 317-346.

[16] I. Karatzas, J. Lehoczky, S. Sethi, And S. Shreve, Explicit solution of a general investmentconsumption problem, Math. Oper. Res., 11 (1986), pp. 261-294.

[17] I. Karatzas And S. Shreve, Brownian Motion and Stochastic Calculus, Springer-Verlag, New York, 1988.

[18] T. G. Kurtz, Random time changes and convergence in distribution under the Meyer-Zheng conditions, Ann. Probab., 19 (1991), pp. 1010-1034. 
[19] H. J. Kushner And P. Dupuis, Numerical Methods for Stochastic Control Problems in Continuous Time, 2nd ed., Springer-Verlag, New York, 2001.

[20] H. J. Kushner And L. F. MARTins, Numerical methods for stochastic singular control problems, SIAM J. Control Optim., 29 (1991), pp. 1443-1475.

[21] L. F. Martins And H. J. Kushner, Routing and singular control for queueing networks in heavy traffic, SIAM J. Control Optim., 28 (1990), pp. 1209-1233.

[22] L. F. Martins, S. E. Shreve, and H. M. Soner, Heavy traffic convergence of a controlled, multi-class queuing system, SIAM J. Control Optim., 34 (1996), pp. 2133-2171.

[23] H. LiU, Optimal consumption and investment with transaction costs and multiple risky assets, J. Finance, 59 (2004), pp. 289-338.

[24] M. J. P. Magill and G. M. Constantinides, Portfolio selection with transaction costs, J. Econom. Theory, 13 (1976), pp. 245-263.

[25] R. C. Merton, Optimum consumption and portfolio rules in a continuous-time model, J. Econom. Theory, 3 (1971), pp. 373-413.

[26] J. L. Menaldi And M. Robin, On some cheap control problems for diffusion processes, Trans. Amer. Math. Soc., 278 (1983), pp. 771-802.

[27] P. A. Meyer And W. A. Zheng, Tightness criteria for laws of semimartingales, Ann. Inst. H. Poincaré Probab. Statist., 20 (1984), pp. 353-372.

[28] P. Protter, Stochastic Integration and Differential Equations, 2nd ed., Springer-Verlag, New York, 2004.

[29] S. E. Shreve And H. M. Soner, Optimal investment and consumption with transaction costs, Ann. Appl. Probab., 4 (1991), pp. 609-692.

[30] P. E. Souganidis, Approximation schemes for viscosity solutions of Hamilton-Jacobi equations, J. Differential Equations, 56 (1985), pp. 345-390.

[31] A. TOURIN AND T. ZARIPhOPOUlou, Viscosity solutions and numerical schemes for investment/consumption models with transaction costs, in Numerical Methods in Finance, Cambridge University Press, Cambridge, 1997, pp. 245-269. 
Copyright of SIAM Journal on Control \& Optimization is the property of Society for Industrial and Applied Mathematics and its content may not be copied or emailed to multiple sites or posted to a listserv without the copyright holder's express written permission. However, users may print, download, or email articles for individual use. 